\title{
ON THE CLASSIFICATION OF NUCLEAR C*-ALGEBRAS
}

\author{
MARIUS DADARLAT AND SØREN EILERS
}

\section{Introduction}

Two of the most influential works on $\mathrm{C}^{*}$-algebras from the mid-seventies Brown, Douglas and Fillmore's [6] and Elliott's [21] - both contain uniqueness and existence results in the now standard sense which we shall outline below. These papers served as keystones for two separate theories - KK-theory and the classification program - which for many years parted ways with only moderate interaction. But with this common origin in mind, it is not surprising that recent years have seen a fruitful interaction which has been one of the main engines behind rapid progress in the classification program.

In the present paper we take this interaction even further. Combining a concept of quasidiagonality for representations and a new characterization of equivalence in the Cuntz picture of $K K$-theory, we achieve a general uniqueness result. And from the quasidiagonality notion we derive a corresponding general existence result by comparing well-known realizations of the $K K$-groups. These results are then employed to obtain new classification results for certain classes of quasidiagonal $\mathrm{C}^{*}$-algebras introduced by $\mathrm{H}$. Lin. An important novel feature of these classes is that they are defined by a certain local approximation property, rather than by an inductive limit construction.

We emphasize that our fundamental uniqueness result does not depend on the universal coefficient theorem (UCT), nor on nuclearity of the $\mathrm{C}^{*}$-algebras involved. On the other hand, when we refine the uniqueness result for use in classification, we need to gradually add such conditions on the $\mathrm{C}^{*}$-algebras. It is to be expected that requirements of this type are necessary, but we have found that holding them back as long as possible leads to more conceptual proofs while clarifying the role of the UCT and nuclearity in classification. Further, since uniqueness results answer fundamental questions about $K K$ - and $K$-theory, they are of interest in themselves. We have pursued this theme in [14], and defer to this paper the proof of the new characterization of $K K$-theory which lies behind our uniqueness results.

Our existence and uniqueness results are in the spirit of the classical Ext-theory from [6]. The main complication overcome in the paper is to control the stabilization which is necessary when one works with finite $\mathrm{C}^{*}$-algebras. In the infinite case, where programs of this type have already been successfully carried out, stabilization is unnecessary. Yet, our methods are sufficiently versatile to allow us to reprove, from a handful of basic results, the classification of purely infinite nuclear $\mathrm{C}^{*}$-algebras of Kirchberg and Phillips.

Received 1 October 1999; revised 7 October 2001.

2000 Mathematics Subject Classification 46L35 (primary), 19K14, 19K35, 46L80 (secondary).

The first author was partially supported by the National Science Foundation, grant numbers 9622434-DMS and 9970223-DMS. The second author was partially supported by the Carlsberg Foundation. 
Indeed, it is our hope that this can be the starting point of a unified approach to the classification of nuclear $\mathrm{C}^{*}$-algebras.

\subsection{Existence and uniqueness}

Existence and uniqueness theorems can be found in most, if not all, classification papers to combine with refinements of Elliott's intertwining argument to yield classification theorems.

In this framework, a uniqueness result allows one to conclude that if the $K$ theoretical elements induced by two *-homomorphisms $\varphi, \psi: A \rightarrow B$ coincide, where $A$ and $B$ are $C^{*}$-algebras satisfying extra conditions, then $\varphi$ and $\psi$ are equivalent in a sense which generalizes unitary equivalence. In [21], one concludes from the fact that $\varphi_{*}=\psi_{*}$ on $K_{0}(A)$ that $\varphi$ and $\psi$ are approximately unitarily equivalent when $A$ and $B$ are both approximately finite-dimensional (AF, cf. [4]). In [6] one shows that if $[\varphi]_{K K}=[\psi]_{K K}$, then $\varphi$ and $\psi$ are unitarily equivalent whenever $A=C(X)$ and $B=\mathscr{L}(H) / \mathscr{K}(H)$.

The role of the equally important existence results is to provide means of realizing given $K$-theoretical data by a sufficiently multiplicative completely positive contractive map. In [21], one realizes any positive element of $\operatorname{Hom}\left(K_{0}(A), K_{0}(B)\right)$ by a $*$-homomorphism when $A$ and $B$ are AF. In [6], any element of $K K(C(X), \mathscr{L}(H) / \mathscr{K}(H))$ is realized by a $*$-monomorphism.

One of the main obstacles in achieving general existence and uniqueness results has been the fact that, as soon as one ventures beyond these classical examples, one only can expect to achieve stable versions of such theorems in a sense that involves adding or subtracting maps of the form $\mu: A \rightarrow \mathbf{M}_{n}(B)$. The challenge has been to control the complexity of the stabilization, in order to be able to incorporate these versions into classification results.

This has been quite successfully carried out, using maps $\mu$ with finitedimensional images, in important classes of quasidiagonal $\mathrm{C}^{*}$-algebras. But in most existence and uniqueness results so far the source $A$ has been required to be a member of a small class of $\mathrm{C}^{*}$-algebras forming building blocks for the class of $\mathrm{C}^{*}$-algebras one has tried to classify, leading to restrictions on the ensuing classification results.

We offer, in the present paper, existence and uniqueness results valid for sources way beyond even the full class of nuclear quasidiagonal $C^{*}$-algebras. More precisely, we consider a unital separable source $A$ and a unital $\operatorname{target} B$, and require that this pair allows a nuclearly absorbing and quasidiagonal unital representation $\gamma: A \rightarrow M(\mathscr{K}(H) \otimes B)$ as defined in $\S 2$ below. Such a map exists automatically when $A$ is quasidiagonal, or by Theorem 2.22 whenever $A$ can be embedded in $B$ such that each non-zero $a \in A$ generates a dense ideal of $B$. In this case, our uniqueness result, Theorem 4.5 , states that if $\varphi, \psi: A \rightarrow B$ are two nuclear $*$-homomorphisms inducing the same $K K_{\text {nuc }}$-class, we may conclude that $\varphi$ is stably approximately unitarily equivalent to $\psi$ in a sense involving adding 'finite pieces' of $\gamma$.

As mentioned above, our fundamental uniqueness result does not depend on the universal coefficient theorem of [47], nor on nuclearity of the $\mathrm{C}^{*}$-algebras involved. For a nuclear source algebra $A$ which satisfies the UCT, our result is predated by one of a similar nature, valid when $A$ or the target algebra is simple, which appears in [34]. However, the approach in [34] had the drawback of relying on a certain result of [50] whose proof was not complete at the time. 
In fact, we shall require uniqueness results which hold also for sufficiently multiplicative completely positive contractive maps, and this entails the problem of associating to such maps a kind of partial $K K$-elements to substitute for the globally defined group homomorphisms one gets from *-homomorphisms. This is done using the universal multicoefficient theorem of [19] in a fashion explained by the first named author in his talk at the Workshop on the classification of amenable $C^{*}$-algebras at the Fields Institute in December of 1994. As soon as we have made sense out of this concept, a uniqueness result for completely positive contractive maps, Theorem 4.15 , can be derived from those for $*$-homomorphisms using a procedure originating with [37] in the torsion-free case. This method also requires keeping a close eye on the $K$-theory for products of $\mathrm{C}^{*}$-algebras.

In our existence result Theorem 5.5 we manage to realize - partially, in a sense corresponding to the one used in the uniqueness result - a given element from $K K_{\text {nuc }}(A, B)$ as a difference of nuclear completely positive contractive maps from $A$ to $\mathbf{M}_{N}(B)$. Again, all we require is that there exists a strictly nuclear nuclearly absorbing quasidiagonal representation $\gamma: A \rightarrow M(\mathscr{K}(H) \otimes B)$. Furthermore, one of the maps in the difference can be chosen as a 'finite piece' of $\gamma$.

In the building block approach, establishing existence has typically been somewhat easier than achieving uniqueness results. This may still be the case in our setting, but at the current stage it is in fact the existence which is causing problems. Indeed, the existence result offered in our paper has shortcomings in the finite case which are responsible for a number of unwanted, and hopefully redundant, restrictions in the resulting classification results. For instance, we do not have sufficient technology to prove in full generality that a positive $K K$-element can be realized by a single map, as one would expect to be the case.

As a main application, we apply our existence and uniqueness results to the class of tracially $\mathrm{AF} \mathrm{C}^{*}$-algebras introduced and studied by $\mathrm{H}$. Lin. Using a factorization property of these $\mathrm{C}^{*}$-algebras, we are able to prove in Theorem 6.12 that, up to an isomorphism, there is only one unital, separable, nuclear and simple tracially $\mathrm{AF} \mathrm{C}^{*}$-algebra satisfying the UCT with $K_{0}(A)=\mathbb{Q}$ and $K_{1}(A)=G$, where $G$ is a countable fixed arbitrary group, thus proving that every such $\mathrm{C}^{*}$-algebra falls in the well-studied class of $A D$-algebras of real rank 0 .

We believe, however, that our existence and uniqueness results will be applicable to a wide range of classification problems, extending well beyond the tracially $\mathrm{AF}$ case. In fact, it might be that at least the uniqueness result will be sufficiently versatile to serve as a unifying element for many future efforts to classify nuclear $\mathrm{C}^{*}$-algebras. To substantiate this claim, we apply our methods to the case of purely infinite $\mathrm{C}^{*}$-algebras, reproving in Theorem 6.21 , rather easily, the classification theorem of purely infinite simple unital nuclear $\mathrm{C}^{*}$-algebras (see [30] and [41]) from a handful of fundamental results about such algebras.

We reported on the present paper at the 1998 Great Plains Operator Theory Seminar. At the same conference, $\mathrm{H}$. Lin reported results which - although the methods differ overlap with our classification results in the tracially $\mathrm{AF} \mathrm{C}^{*}$-algebra case. More details are given in the notes of the present paper. The exposition of the paper, and many intermediate results, have been improved since the first final version as regards the role of nuclearity as described above. However, the end results are the same.

We owe tremendous thanks to Takeshi Katsura who read a previous version of the paper, pointed out a number of errors, and suggested corrections. 


\subsection{Methods}

Apart from $K K$-theory, the main technical tools are approximate morphisms and partial $K K$-elements, defined using $K$-theory with coefficients.

We arrive at uniqueness and existence results from the existence of an absorbing and quasidiagonal representation. To achieve such representations, one may use the Kasparov Weyl-von Neumann-Voiculescu theorem, along with the concept of quasidiagonality. One can also employ a related result by $\mathrm{H}$. Lin [34] stating that a certain representation associated to a unital inclusion $\iota: A \rightarrow B$ is absorbing when $A$ is nuclear and $B$ is simple. A generalization of this result is offered in Theorem 2.22 .

To refine uniqueness results we depend on a number of basic properties about the $K$-theory of products of $\mathrm{C}^{*}$-algebras. Furthermore, to apply our results to classify tracially AF $\mathrm{C}^{*}$-algebras, we use several results by Lin about structural properties of such $\mathrm{C}^{*}$-algebras. We prove classification for purely infinite algebras by appealing to the embedding theorem for exact $\mathrm{C}^{*}$-algebras of [31], as well as structural results by Kirchberg, Phillips and Rørdam.

\section{Absorbing and quasidiagonal representations}

In the paper, a representation refers to a $*$-homomorphism from a general $\mathrm{C}^{*}$-algebra to a $\mathrm{C}^{*}$-algebra of the form $\mathscr{L}(E)$, where $E$ is a Hilbert $\mathrm{C}^{*}$-algebra module. The reader is referred to [29] for an introduction to these objects. Throughout the paper, all Hilbert $\mathrm{C}^{*}$-algebra modules are assumed to be countably generated over a $\sigma$-unital $\mathrm{C}^{*}$-algebra $B$. As usual, the canonical such module is denoted by $H_{B}$.

In this section we are going to define two properties for representations of a $\mathrm{C}^{*}$-algebra which are refinements of known properties. Of these notions, the definition of nuclearly absorbing representations is going to ensure that a given representation, in a sense to be made precise, contains the full representation theory of the $\mathrm{C}^{*}$-algebra, whereas the quasidiagonality property ensures that the representation can be approximated by finite-dimensional compressions. These two properties combine to allow uniqueness and existence results in the following.

\subsection{Two properties of representations}

Definition 2.1. Fix a $\sigma$-unital $\mathrm{C}^{*}$-algebra $B$. When $\gamma: A \rightarrow \mathscr{L}(E)$ and $\gamma^{\prime}: A \rightarrow \mathscr{L}\left(E^{\prime}\right)$ are two representations, with $E$ and $E^{\prime}$ Hilbert $B$-modules, we say that $\gamma$ and $\gamma^{\prime}$ are approximately unitarily equivalent, and write $\gamma \sim \gamma^{\prime}$, if there exists a sequence of unitaries $u_{n} \in \mathscr{L}\left(E^{\prime}, E\right)$ such that

(i) $\lim _{n \rightarrow \infty}\left\|\gamma(a)-u_{n} \gamma^{\prime}(a) u_{n}^{*}\right\|=0$,

(ii) $\gamma(a)-u_{n} \gamma^{\prime}(a) u_{n}^{*} \in \mathscr{K}(E)$

for any $a \in A$.

We say that a representation $\gamma$ is absorbing if $\gamma \sim \gamma \oplus \sigma$ for any $\sigma$. We now give a generalization of that notion.

Definition 2.2 [51, Definition 1.1.d]. A completely positive contraction $\pi: A \rightarrow \mathscr{L}(E)$ is said to be strictly nuclear if there exist integers $\left(n_{\lambda}\right)$ and 
generalized sequences $\psi_{\lambda}: A \rightarrow M_{n_{\lambda}}$ and $\varphi_{\lambda}: M_{n_{\lambda}} \rightarrow \mathscr{L}(E)$ of completely positive contractions such that

$$
\lim _{\lambda \rightarrow \infty} \varphi_{\lambda} \psi_{\lambda}(a)=\pi(a)
$$

in the strict topology, for all $a \in A$.

REMARK 2.3. If either $A$ or $B$ is nuclear, then any completely positive contraction $\pi: A \rightarrow \mathscr{L}(E)$ is strictly nuclear, by [51, 1.7]. Any scalar representation $\theta: A \rightarrow \mathscr{L}(H) \subset \mathscr{L}\left(H_{B}\right)$ is strictly nuclear, since it factors through a module over the nuclear $\mathrm{C}^{*}$-algebra $\mathbb{C}$.

Definition 2.4. A non-unital representation $\pi: A \rightarrow \mathscr{L}(E)$ is called nuclearly absorbing if $\pi \oplus \sigma \sim \pi$ for any strictly nuclear representation $\sigma: A \rightarrow \mathscr{L}(F)$. A unital representation $\pi: A \rightarrow \mathscr{L}(E)$ is called nuclearly absorbing if $\pi \oplus \sigma \sim \pi$ for any unital strictly nuclear representation $\sigma: A \rightarrow \mathscr{L}(F)$.

We emphasize that we have two different versions of nuclearly absorbing representations, one for unital representations and a different one for non-unital representations. We are going to explain how they interrelate in Lemma 2.17 below.

Definition 2.5. A representation $\gamma: A \rightarrow M(\mathscr{K}(H) \otimes B)$ is quasidiagonal if there exists an approximate unit of projections $\left(e_{n}\right)$ for $\mathscr{K}(H) \otimes B$ with the property that

$$
\left[e_{n}, \gamma(a)\right] \rightarrow 0,
$$

for any $a \in A$. It is clear that if $\gamma$ is quasidiagonal, one may assume that $\left(e_{n}\right)$ has the property that $e_{n} \in \mathbf{M}_{r_{n}}(B)$ for some sequence of integers $\left(r_{n}\right)$. When this is the case, we define a sequence of completely positive maps $\gamma_{n}: A \rightarrow \mathbf{M}_{r_{n}}(B)$ by $\gamma_{n}(a)=e_{n} \gamma(a) e_{n}$. We call $\left(\gamma_{n}\right)_{n \in \mathbb{N}}$ a quasidiagonalization of $\gamma$ by $\left(e_{n}\right)_{n \in \mathbb{N}}$.

The concept of relative quasidiagonality that we use in this paper was studied in $[48]$ and $[50]$.

REMARK 2.6. (i) One notes that a nuclearly absorbing representation need not be strictly nuclear.

(ii) If a representation is both strictly nuclear and quasidiagonal, then it follows from [51] that any element of a quasidiagonalization is a nuclear map. Indeed, $\pi$ is strictly nuclear precisely when for any $v \in \mathscr{K}(E)$, the map $A \rightarrow \mathscr{K}(E)$, defined by $a \mapsto v^{*} \pi(a) v$, is nuclear.

(iii) If $\gamma$ is quasidiagonal and $\gamma \sim \gamma^{\prime}$, then $\gamma^{\prime}$ is quasidiagonal. Thus if there exists a strictly nuclear nuclearly absorbing and quasidiagonal representation $\gamma: A \rightarrow M(\mathscr{K}(H) \otimes B)$, then any strictly nuclear nuclearly absorbing representation will be quasidiagonal.

\subsection{Fundamental examples}

Definition 2.7. Let $A$ be a separable $C^{*}$-algebra. An admissible scalar representation $\theta: A \rightarrow M(\mathscr{K}(H) \otimes B)$ is a $*$-homomorphism which factors as

$$
A \stackrel{\theta^{\prime}}{\longrightarrow} \mathscr{L}(H) \stackrel{-\otimes 1}{\longrightarrow} \mathscr{L}(H) \otimes M(B) \longleftrightarrow M(\mathscr{K}(H) \otimes B)
$$


where $\theta^{\prime}$ is faithful and of infinite multiplicity, that is, of the form $\infty \cdot \gamma$ for some representation $\gamma$.

Definition 2.8. A unital *-homomorphism $\iota: A \hookrightarrow B$ is called a full embedding if $\operatorname{span} B \iota(a) B$ is dense in $B$ for all non-zero $a \in A$.

The following definition gives a class of examples of full embeddings.

Definition 2.9. We say that a unital $*$-homomorphism $\iota: A \rightarrow B$ is a unital simple embedding if it can be factored

$$
A \stackrel{\iota^{\prime}}{\longrightarrow} C \stackrel{\iota^{\prime \prime}}{\longrightarrow} B
$$

where $\iota^{\prime}$ and $\iota^{\prime \prime}$ are injective and $C$ is simple.

This implies that $C, \iota^{\prime}$ and $\iota^{\prime \prime}$ are unital. Note that the composition (on either side) of a simple embedding with a unital injective $*$-homomorphism is a simple embedding. In this setting, we define a representation $d_{l}: A \rightarrow M(\mathscr{K}(H) \otimes B)$ by

$$
d_{\iota}(a)=1 \otimes \iota(a)
$$

With all the definitions in place we can now state the main results of the ensuing sections as follows. Let $A$ be a unital separable $\mathrm{C}^{*}$-algebra.

(i) If $\theta: A \rightarrow \mathscr{L}\left(H_{B}\right)$ is a unital admissible scalar representation, then $\theta$ is nuclearly absorbing (Proposition 2.18).

(ii) If $\iota: A \hookrightarrow B$ is a unital full embedding, then $d_{\iota}$ is nuclearly absorbing (Theorem 2.22).

Here (i) follows from the arguments of [29], as pointed out in [51]. As we need many of the elements involved in proving this, we are going to supply details for the benefit of the reader. Further, (ii) is a generalization of a result in [34], where it was proved for $\iota: A \rightarrow B$ a unital embedding, with $A$ nuclear, and either $A$ or $B$ simple.

The results combine with the quasidiagonality condition. Indeed, note that $d_{\iota}$ is always quasidiagonal, as it commutes with projections $e_{n}=n \cdot 1_{B}$. Thus by Remark 2.6(iii) all unital strictly nuclear nuclearly absorbing representations $\gamma: A \rightarrow M(\mathscr{K}(H) \otimes B)$ are quasidiagonal when there is a unital nuclear full embedding of $A$ into $B$.

If $A$ is a quasidiagonal $C^{*}$-algebra, then by definition, it has an admissible scalar representation $\theta: A \rightarrow M(\mathscr{K}(H) \otimes B)$ which is quasidiagonal. Therefore any strictly nuclear nuclearly absorbing representation of $A$ is quasidiagonal. We are going to need the following subclass of the quasidiagonal $\mathrm{C}^{*}$-algebras.

Definition 2.10. Let $A$ be a separable $\mathrm{C}^{*}$-algebra and let $\left(\pi_{n}\right)$ be a sequence of finite-dimensional representations of $A$. We say that $\left(\pi_{n}\right)$ is a separating sequence if for every $a \in A$ there is $n$ such that $\pi_{n}(a) \neq 0$. If this is the case, we say that $A$ is residually finite dimensional or RFD.

Finally, note that, as in Remark 2.3, the map $\theta$ is always strictly nuclear. The map $d_{\iota}$ is strictly nuclear precisely when $\iota$ is nuclear; cf. Remark 2.6(ii). 


\subsection{Absorbing representations (after Kasparov)}

Definition 2.11. If $\pi: A \rightarrow \mathscr{L}(E)$ is a representation and $\varphi: A \rightarrow \mathscr{K}(F)$ is a completely positive map, we write $\varphi<\pi$ if there is a bounded sequence $v_{i} \in \mathscr{K}(F, E)$ such that

(i) $\lim _{i \rightarrow \infty}\left\|\varphi(a)-v_{i}^{*} \pi(a) v_{i}\right\|=0$ for all $a \in A$,

(ii) $\lim _{i \rightarrow \infty}\left\|v_{i}^{*} \xi\right\|=0$ for all $\xi \in E$.

The condition (ii) above is equivalent to

(ii') $\lim _{i \rightarrow \infty}\left\|v_{i}^{*} x\right\|=0$ for all $x \in \mathscr{K}(E)$.

If $\sigma: A \rightarrow \mathscr{L}(F)$ is a representation, we define $\sigma_{\infty}: A \rightarrow \mathscr{L}\left(F_{\infty}\right)$ by $\sigma_{\infty}=\sigma \oplus \sigma \oplus \ldots$, where $F_{\infty}=F \oplus F \oplus \ldots$.

The proof of the following lemma is routine; we leave it to the reader.

Lemma 2.12. Let $\pi$ and $\pi^{\prime}$ be representations of $A$ on $\mathscr{L}(E)$ and $\mathscr{L}\left(E^{\prime}\right)$, and let $\varphi$ and $\varphi^{\prime}$ be completely positive maps from $A$ to $\mathscr{K}(F)$ and $\mathscr{K}\left(F^{\prime}\right)$. We have the following.

(i) If $\varphi<\pi$ and $\varphi^{\prime}<\pi$, then $\varphi \oplus \varphi^{\prime}<\pi$.

(ii) If $\varphi<\pi$, then $\varphi<\pi \oplus \pi^{\prime}$.

(iii) If $\varphi<\pi$, and $\pi \sim \pi^{\prime}$, then $\varphi<\pi^{\prime}$.

(iv) If $v \in \mathscr{K}(E)$, then $v \pi(\cdot) v^{*}<\pi_{\infty}$.

We can now state the main result of this section.

THeOREM 2.13. Let $A$ be a unital separable $C^{*}$-algebra and let $B$ be a $\sigma$-unital $C^{*}$-algebra. Let $\pi: A \rightarrow \mathscr{L}(E)$ and $\sigma: A \rightarrow \mathscr{L}(F)$ be two unital representations where $E$ and $F$ are Hilbert B-modules. The following assertions are equivalent:

(i) $\pi \sim \sigma_{\infty} \oplus \pi$;

(ii) for any $v \in \mathscr{K}(F)$, if $\varphi=v^{*} \sigma(\cdot) v$, then $\varphi<\pi$;

(iii) there is an increasing approximate unit $\left(e_{n}\right)$ of $\mathscr{K}(F)$, with $e_{1}=0$ and quasicentral in $\sigma(A)+\mathscr{K}(F)$, such that

$$
\left(e_{n+k}-e_{n}\right)^{1 / 2} \sigma(\cdot)\left(e_{n+k}-e_{n}\right)^{1 / 2}<\pi
$$

for all $n, k \geqslant 1$.

The proof of the theorem, given at the end of the section, is based on the following three results, due essentially to Kasparov [29].

Lemma 2.14. Let $A$ and $B$ be as in Theorem 2.13, and let $\sigma: A \rightarrow \mathscr{L}(F)$ be a representation. Let $\left(e_{n}\right)$ be an increasing approximate unit of $\mathscr{K}(F)$, quasicentral in $\sigma(A)+\mathscr{K}(F)$. Then for any compact subset $\mathscr{F} \subset A$ and any $\varepsilon>0$ there is a subsequence $\left(e_{n(k)}\right)$ of $\left(e_{n}\right)$ such that if $f_{1}=e_{n(2)}^{1 / 2}$ and $f_{k}=\left(e_{n(k+1)}-e_{n(k)}\right)^{1 / 2}$ for $k \geqslant 2$, then

(i) $\lambda(a)=\sum_{k=1}^{\infty} f_{k} \sigma(a) f_{k}$ is strictly convergent, for all $a \in A$,

(ii) $\sigma(a)-\lambda(a) \in \mathscr{K}(F)$ for all $a \in A$,

(iii) $\|\sigma(a)-\lambda(a)\|<\varepsilon$ for all $a \in \mathscr{F}$. 
Proof. This is a reformulation of [29, Lemma 10].

Proposition 2.15. Let $A, B, \pi: A \rightarrow \mathscr{L}(E)$ and $\sigma: A \rightarrow \mathscr{L}(F)$ be as in Theorem 2.13, satisfying condition (iii) there. Then there is a sequence of isometries $v_{i} \in \mathscr{L}(F, E)$ such that

(i) $v_{i} \sigma(a)-\pi(a) v_{i} \in \mathscr{K}(F, E)$,

(ii) $\lim _{i \rightarrow \infty}\left\|v_{i} \sigma(a)-\pi(a) v_{i}\right\|=0$ for all $a \in A$.

Proof. The result is implicit in the proof of [29, Theorem 5] and the remark following it. We outline the proof for the benefit of the reader. Since $\sigma$ is a *-homomorphism, it suffices to find a sequence of isometries $v_{i}$ with

$$
\lim _{i \rightarrow \infty}\left\|\sigma(a)-v_{i}^{*} \pi(a) v_{i}\right\|=0, \quad \text { and } \quad \sigma(a)-v_{i}^{*} \pi(a) v_{i} \in \mathscr{K}(F),
$$

for each $a \in A$. Indeed, as in [2], this follows from the identity

$$
\begin{aligned}
\left(v_{i} \sigma(a)-\right. & \left.\pi(a) v_{i}\right)^{*}\left(v_{i} \sigma(a)-\pi(a) v_{i}\right) \\
= & -\left(\sigma\left(a^{*} a\right)-v_{i}^{*} \pi\left(a^{*} a\right) v_{i}\right)+\left(\sigma\left(a^{*}\right)-v_{i}^{*} \pi\left(a^{*}\right) v_{i}\right) \sigma(a) \\
& +\sigma\left(a^{*}\right)\left(\sigma(a)-v_{i}^{*} \pi(a) v_{i}\right) .
\end{aligned}
$$

Fix $\varepsilon>0$ and $\mathscr{F} \subset A$ a compact subset with dense span, such that $1_{A} \in \mathscr{F}=\mathscr{F}^{*}$. We need to find an isometry $v \in \mathscr{L}(F, E)$ such that

$$
\left\|\sigma(a)-v^{*} \pi(a) v\right\|<h(\varepsilon) \text { and } \sigma(a)-v^{*} \pi(a) v \in \mathscr{K}(F)
$$

for all $a \in \mathscr{F}$. Here $h$ is a universal function with $\lim _{\varepsilon \rightarrow 0} h(\varepsilon)=0$.

Let $\left(e_{n}\right)$ be as in condition (iii) of Theorem 2.13. Let $\lambda(a)=\sum_{k=1}^{\infty} f_{k} \sigma(a) f_{k}$ be given by Lemma 2.14 . Using condition (iii), we find inductively a sequence $z_{k} \in \mathscr{K}(F, E)$ such that for all $a \in \mathscr{F}$ and $j, k$,

$$
\begin{aligned}
\left\|f_{k} \sigma(a) f_{k}-z_{k}^{*} \pi(a) z_{k}\right\| & \leqslant \varepsilon \cdot 2^{-k}, \\
\left\|z_{k}^{*} \pi(a) z_{j}\right\| & \leqslant \varepsilon \cdot 2^{-j-k} \quad \text { for } j<k, \\
\left\|z_{k}^{*} g_{j}\right\| & \leqslant 2^{-k} \quad \text { for } j \leqslant k,
\end{aligned}
$$

where $\left(g_{j}\right)$ is a countable approximate unit of $\mathscr{K}(F)$. Then, as in the proof of [29, Theorem 5], one shows that $\sum_{k=1}^{\infty} z_{k}$ converges strictly to an element $z \in \mathscr{L}(F, E)$ satisfying

$$
\left\|\sigma(a)-z^{*} \pi(a) z\right\|<3 \varepsilon \quad \text { and } \quad \sigma(a)-z^{*} \pi(a) z \in \mathscr{K}(F)
$$

for all $a \in \mathscr{F}$. Since $\sigma(1)=\pi(1)=1$ and $1 \in \mathscr{F},\left\|z^{*} z-1\right\|<3 \varepsilon$. To obtain (2.1), one replaces $z$ by the isometry $v=z\left(z^{*} z\right)^{-1 / 2}$.

Let $w_{\infty}: F_{\infty} \rightarrow F \oplus F_{\infty}$ be defined as $w_{\infty}\left(\xi_{1}, \xi_{2}, \xi_{3}, \ldots\right)=\xi_{1} \oplus\left(\xi_{2}, \xi_{3}, \ldots\right)$.

Lemma 2.16. Let $\pi: A \rightarrow \mathscr{L}(E)$ and $\sigma: A \rightarrow \mathscr{L}(F)$ be two representations. Then for any isometry $v: F_{\infty} \rightarrow E$, the unitary

$$
u=\left(1_{F} \oplus v\right) w_{\infty} v^{*}+1_{E}-v v^{*} \in \mathscr{L}(E, F \oplus E)
$$

satisfies

$$
\left\|\sigma(a) \oplus \pi(a)-u \pi(a) u^{*}\right\| \leqslant 6\left\|v \sigma_{\infty}(a)-\pi(a) v\right\|+4\left\|v \sigma_{\infty}\left(a^{*}\right)-\pi\left(a^{*}\right) v\right\| .
$$


Moreover, if $v \sigma_{\infty}(a)-\pi(a) v \in \mathscr{K}\left(F_{\infty}, E\right)$, for $a \in A$, then

$$
\sigma(a) \oplus \pi(a)-u \pi(a) u^{*} \in \mathscr{K}(F \oplus E),
$$

for $a \in A$.

Proof. Although the proof of the lemma is implicit in the proof of [29, Theorem 6], we supply a complete argument. Note that the two summands of $u$ are partial isometries. If $p=v v^{*}$ and $p^{\perp}=1_{E}-p$, then $\left(1_{F} \oplus v\right) w_{\infty} v^{*}: p E \rightarrow F \oplus p E$ and $p^{\perp}: p^{\perp} E \rightarrow p^{\perp} E$ act as unitaries. Abbreviate $\omega(a)=\left\|v \sigma_{\infty}(a)-\pi(a) v\right\|$.

First assume that $\pi(a)$ and $p$ commute. We have

$$
\begin{aligned}
u \pi(a) u^{*} & =\left(1_{F} \oplus v\right) w_{\infty} v^{*} p \pi(a) p v w_{\infty}^{*}\left(1_{F} \oplus v^{*}\right) \oplus p^{\perp} \pi(a) p^{\perp} \\
& \approx_{\omega}\left(1_{F} \oplus v\right) w_{\infty} \sigma_{\infty}(a) w_{\infty}^{*}\left(1_{F} \oplus v^{*}\right) \oplus p^{\perp} \pi(a) p^{\perp} \\
& =\sigma(a) \oplus v \sigma_{\infty}(a) v^{*} \oplus p^{\perp} \pi(a) p^{\perp} \\
& \approx_{\omega} \sigma(a) \oplus p \pi(a) p \oplus p^{\perp} \pi(a) p^{\perp} \\
& =\sigma(a) \oplus \pi(a),
\end{aligned}
$$

where ' $\approx_{\omega}$ ' indicates that the error is dominated in norm by $\omega(a)$ and that it is compact if $v \sigma_{\infty}(a)-\pi(a) v$ is.

For general $\pi(a)$, we consider instead

$$
\pi^{\prime}(a)=p \pi(a) p \oplus p^{\perp} \pi(a) p^{\perp} .
$$

Note that $\omega^{\prime}(a)=\left\|v \sigma_{\infty}(a)-\pi^{\prime}(a) v\right\| \leqslant \omega(a)$ and that $v \sigma_{\infty}(a)-\pi^{\prime}(a) v$ is compact under the compactness assumption on $v$. We have $\left\|\sigma_{\infty}(a)-v^{*} \pi(a) v\right\| \leqslant \omega(a)$, so that

$$
\begin{aligned}
\left\|p^{\perp} \pi(a) p\right\| & \leqslant\|\pi(a) p-p \pi(a)\| \\
& \leqslant\left\|\pi(a) v v^{*}-v \sigma_{\infty}(a) v^{*}\right\|+\left\|v \sigma_{\infty}(a) v^{*}-v v^{*} \pi(a)\right\| \\
& \leqslant \omega(a)+\omega\left(a^{*}\right)
\end{aligned}
$$

it is also clear that $p^{\perp} \pi(a) p$ is compact if $v \sigma_{\infty}(a)-\pi(a) v$ is. This completes the proof by reducing it to the first case, up to an error of $4\left(\omega(a)+\omega\left(a^{*}\right)\right)$ which is compact under the compactness assumption on $v$.

Proof of Theorem 2.13. Using three observations from Lemma 2.12 we get (i) $\Rightarrow$ (ii) via

$$
\varphi=v^{*} \sigma(\cdot) v<\sigma_{\infty} \oplus \pi \sim \pi .
$$

If $\varphi=v^{*} \sigma(\cdot) v<\sigma_{\infty}$, then $\varphi<\sigma_{\infty} \oplus \pi$; hence $\varphi<\pi$ by Lemma 2.12(iii). The implication (ii) $\Rightarrow$ (iii) is obvious; take $\left(e_{n}\right)$ to be any countable approximate unit of $\mathscr{K}(F)$, quasicentral in $\sigma(A)+\mathscr{K}(F)$ (cf. [2]). To prove (iii) $\Rightarrow$ (i) we first notice that if $\sigma, \pi$ and $e_{n} \in \mathscr{K}(F)$ satisfy (iii), then so do $\sigma_{\infty}, \pi$ and

$$
e_{n}^{\prime}=\underbrace{e_{n} \oplus \ldots \oplus e_{n}}_{n} \oplus 0 \oplus \ldots \in \mathscr{K}\left(F_{\infty}\right) \text {, }
$$

by Lemma 2.12(i). Since $\left(\sigma_{\infty}\right)_{\infty}=\sigma_{\infty}$, from Proposition 2.15 we find a sequence of isometries $v_{i} \in \mathscr{L}\left(F_{\infty}, E\right)$ such that

$$
v_{i}\left(\sigma_{\infty}\right)_{\infty}(a)-\pi(a) v_{i} \in \mathscr{K}\left(F_{\infty}, E\right) \quad \text { and } \quad \lim _{i \rightarrow \infty}\left\|v_{i}\left(\sigma_{\infty}\right)_{\infty}(a)-\pi(a) v_{i}\right\|=0
$$


for all $a \in A$. Let $\left(u_{i}\right)$ be a sequence of unitaries given by Lemma 2.16 applied to $\pi, \sigma_{\infty}$ and $v_{i}$. Then

$\sigma_{\infty}(a) \oplus \pi(a)-u_{i} \pi(a) u_{i}^{*} \in \mathscr{K}\left(F_{\infty} \oplus E\right) \quad$ and $\quad \lim _{i \rightarrow \infty}\left\|\sigma_{\infty} \oplus \pi(a)-u_{i} \pi(a) u_{i}^{*}\right\|=0$

for all $a \in A$. Thus $\sigma_{\infty} \oplus \pi \sim \pi$.

\subsection{Nuclearly absorbing representations}

If $\pi: A \rightarrow \mathscr{L}(E)$ is a representation, we denote by $\widetilde{A}$ the $\mathrm{C}^{*}$-algebra obtained by adjoining an external unit $\widetilde{1}$ to $A$, and define $\widetilde{\pi}: \widetilde{A} \rightarrow \mathscr{L}(E)$ by $\widetilde{\pi}(a+\lambda \widetilde{1})=\pi(a)+\lambda 1_{E}$.

A unital representation $\gamma: A \rightarrow M(\mathscr{K}(H) \otimes B)$ cannot absorb a non-unital representation. However, if $\widehat{\gamma}=0 \oplus \gamma: A \rightarrow M(\mathscr{K}(H \oplus H) \otimes B)$, where the zero summand acts on $H \otimes B$, then we have the following result.

Lemma 2.17. Let $A$ be a separable $C^{*}$-algebra and let $B$ be a $\sigma$-unital $C^{*}$-algebra. If $\pi$ is a non-unital nuclearly absorbing representation, then $\tilde{\pi}$ is a unital nuclearly absorbing representation. Suppose that $A$ is unital. If $\gamma: A \rightarrow M(\mathscr{K}(H) \otimes B)$ is a unital nuclearly absorbing representation, then $\widehat{\gamma}$ is a non-unital nuclearly absorbing representation.

Proof. The first part of the lemma is obvious. For the second part, we need to show that if $\varphi: A \rightarrow \mathscr{L}(E)$ is a strictly nuclear representation, then $\varphi \oplus \widehat{\gamma} \sim \widehat{\gamma}$. If $p=\varphi(1)$, then $E \cong p E \oplus p^{\perp} E$ and $\varphi=\varphi_{1} \oplus 0_{p^{\perp} E}$, with $\varphi_{1}=p \varphi(\cdot) p$. We have $\varphi_{1} \oplus \gamma \sim \gamma$, since $\gamma$ is nuclearly absorbing. Since $p^{\perp} E \oplus H_{B} \cong H_{B}$ by [29, Theorem 2], we obtain

$$
\varphi \oplus \widehat{\gamma}=\varphi_{1} \oplus 0_{p^{\perp} E} \oplus 0_{H_{B}} \oplus \gamma \sim \varphi_{1} \oplus \gamma \oplus 0_{H_{B}} \sim 0_{H_{B}} \oplus \gamma=\widehat{\gamma} .
$$

Proposition 2.18. Let $A$ be a unital separable $C^{*}$-algebra. If $\theta: A \rightarrow \mathscr{L}\left(H_{B}\right)$ is a unital admissible scalar representation, then $\theta$ is nuclearly absorbing.

Proof. Given a unital strictly nuclear representation $\sigma: A \rightarrow \mathscr{L}(F)$, we want to show that $\sigma \oplus \theta \sim \theta$. Assume first that $F=H_{B}$. We are going to use the implication (ii) $\Rightarrow$ (i) of Theorem 2.13. If $v \in \mathscr{K}\left(H_{B}\right)$ and $\varphi=v^{*} \sigma(\cdot) v$, we need to show that $\varphi<\theta$. The set of all completely positive maps $\varphi: A \rightarrow \mathscr{K}\left(H_{B}\right)$ with $\varphi<\theta$ is closed in the point-norm topology. Thus, without loss of generality, we may assume that $v \in \mathbf{M}_{n}(B)$ for some $n$. Therefore $\varphi: A \rightarrow \mathbf{M}_{n}(B)$. Since $\varphi$ is a nuclear map, because $\sigma$ is strictly nuclear, $\varphi<\theta$ by [29, Theorem 4]. Hence $\sigma_{\infty} \oplus \theta \sim \theta$ by Theorem 2.13. This implies that $\sigma \oplus \theta \sim \theta$.

The case $F \cong H_{B}$ follows from the previous case, after replacing $\sigma$ by $u \sigma(\cdot) u^{*}$, where $u$ is a unitary implementing the isomorphism $F \cong H_{B}$. Indeed,

$$
\sigma \oplus \theta \sim u \sigma(\cdot) u^{*} \oplus \theta \sim \theta .
$$

If $F \not \equiv H_{B}$, we define $\sigma^{\prime}=\sigma \oplus \theta: A \rightarrow \mathscr{L}\left(F \oplus H_{B}\right)$. Since $F \oplus H_{B} \cong H_{B}$ by [29], $\sigma_{\infty}^{\prime} \oplus \theta \sim \theta$ by the previous part of the proof. Since $\sigma \oplus \sigma_{\infty}^{\prime} \sim \sigma_{\infty}^{\prime}$, we infer that $\sigma \oplus \theta \sim \theta$.

Proposition 2.19. Let $A$ be a unital, separable $C^{*}$-algebra, and let $B$ be a unital $C^{*}$-algebra. Let $\pi: A \rightarrow \mathscr{L}(E)$ be a unital representation. Suppose that 
$\theta: A \rightarrow \mathscr{L}\left(H_{B}\right)$ is a unital admissible scalar representation. Then the following are equivalent:

(i) $\pi$ is nuclearly absorbing;

(ii) $\pi \sim \theta \oplus \pi$;

(iii) for any unital completely positive map $\varphi: A \rightarrow \mathbf{M}_{n}(\mathbb{C}) \subseteq \mathscr{L}\left(B^{n}\right)$, we have $\varphi<\pi$.

Proof. To prove (ii) $\Rightarrow$ (i), let $\sigma: A \rightarrow \mathscr{L}(F)$ be a unital strictly nuclear representation and note that $\theta \oplus \sigma \sim \theta$ by Proposition 2.18. Using (ii) twice, we get

$$
\pi \sim \pi \oplus \theta \sim \pi \oplus \theta \oplus \sigma \sim \pi \oplus \sigma .
$$

The opposite implication is clear since $\theta$ is strictly nuclear, and (ii) $\Rightarrow$ (iii) follows again from [29, Theorem 4], since by Lemma 2.12(iii), $\varphi<\pi \oplus \theta \sim \pi$ implies $\varphi<\pi$. It remains to prove (iii) $\Rightarrow$ (ii), and we shall do so using the implication (iii) $\Rightarrow$ (i) from Theorem 2.13 with $\sigma=\theta=\theta^{\prime} \otimes 1$ with $\theta^{\prime}$ as in Definition 2.7. Embed $\mathbf{M}_{n}(\mathbb{C})$ into $\mathscr{K}(H)$ under an increasing sequence of projections $\left(f_{n}\right)$. There exist a sequence of natural numbers $m(1)<m(2)<\ldots<m(n)<\ldots$ and finite rank operators $x_{n} \in \operatorname{conv}\left\{f_{i}: m(n-1)<i \leqslant m(n)\right\}$ such that $\left(e_{n}\right)=\left(x_{n} \otimes 1_{B}\right)$ is an approximate unit of $\mathscr{K}\left(H_{B}\right)$ which is quasicentral in $\theta(A)+\mathscr{K}\left(H_{B}\right)$. Note that $x_{n} \leqslant f_{m(n)} \leqslant x_{n+1}$. For any $n$ and $i$ we write

with

$$
\left(e_{n+i}-e_{n}\right)^{1 / 2} \theta(\cdot)\left(e_{n+i}-e_{n}\right)^{1 / 2}=\varphi_{n, i}(\cdot) \otimes 1_{B},
$$

$\varphi_{n, i}(\cdot)=\left(x_{n+i}-x_{n}\right)^{1 / 2} \theta^{\prime}(\cdot)\left(x_{n+i}-x_{n}\right)^{1 / 2}=\left(x_{n+i}-x_{n}\right)^{1 / 2} \psi_{n, i}(\cdot)\left(x_{n+i}-x_{n}\right)^{1 / 2}$

where $\psi_{n, i}(\cdot)=f_{m(n+i)} \theta^{\prime}(\cdot) f_{m(n+i)}: A \rightarrow \mathbf{M}_{m(n+i)}(\mathbb{C})$ is a unital completely positive map. Our assumption gives $\psi_{n, i} \otimes 1_{B}<\pi$; hence $\varphi_{n, i} \otimes 1_{B}<\pi$, and so by Theorem 2.13 we conclude that $\pi \oplus \theta_{\infty} \sim \pi$. Hence also $\pi \oplus \theta \sim \pi$.

The following result can be found in [1].

Lemma 2.20. Let $A$ be a unital separable $C^{*}$-algebra and let $\varphi \in P(A)$ be a pure state. Then there is a sequence $\left(x_{n}\right)$ in $A^{+}$with $\left\|x_{n}\right\|=1$ and

for all $a \in A$.

$$
\left\|x_{n}(a-\varphi(a)) x_{n}\right\| \rightarrow 0
$$

A sequence $\left(x_{n}\right)$ with these properties is said to excise the state $\varphi$.

Lemma 2.21. Let $A$ be a unital separable $C^{*}$-algebra and let $\iota: A \hookrightarrow B$ be a unital full embedding. Then $\varphi<d_{\iota}$ for any unital completely positive map $\varphi: A \rightarrow \mathbf{M}_{n}(\mathbb{C}) \subseteq \mathbf{M}_{n}(B)=\mathscr{L}\left(B^{n}\right)$.

Proof. To simplify notation we identify $A$ with $\iota(A)$. Fix a finite set $\mathscr{F} \subseteq A$ and a positive $\varepsilon$. It suffices to find an isometry $V \in \mathscr{L}\left(B^{n}, B^{m}\right)$ with

$$
\left\|\varphi(a)-V^{*}\left(a \otimes 1_{m}\right) V\right\|<\varepsilon
$$

for each $a \in \mathscr{F}$. We may assume that in fact $n=1$ after replacing $\varphi$ by $\bar{\varphi}: \mathbf{M}_{n}(A) \rightarrow B$ as in [29, Lemmas 5, 6], for $\mathbf{M}_{n}(A) \hookrightarrow \mathbf{M}_{n}(B)$ is also a full embedding. 
First assume that $\varphi$ is a pure state. Apply Lemma 2.20 to get an excising sequence $\left(x_{n}\right)$ for $\varphi$, and choose $n_{0}$ such that $x=x_{n_{0}}$ satisfies

$$
\left\|\varphi(a) x^{2}-x a x\right\|<\varepsilon
$$

when $a \in \mathscr{F}$. For every $\eta \in(0,1)$ we define $f_{\eta}, g_{\eta}:[0,1] \rightarrow[0,1]$ by

$$
f_{\eta}(t)=\left\{\begin{array}{ll}
0 & \text { if } t=0, \\
1 & \text { if } t \geqslant \eta, \\
\text { affine } & \text { if } 0 \leqslant t \leqslant \eta,
\end{array} \quad g_{\eta}(t)= \begin{cases}0 & \text { if } t \leqslant \eta, \\
1 & \text { if } t \geqslant \frac{1}{2}(\eta+1), \\
\text { affine } & \text { if } \eta \leqslant t \leqslant \frac{1}{2}(\eta+1) .\end{cases}\right.
$$

Note that for a sufficiently large $\eta_{0}, x=f_{\eta_{0}}\left(x_{n_{0}}\right)$ also satisfies (2.3). Hence if we let $y=g_{\eta_{0}}\left(x_{n_{0}}\right)$, we get a pair of positive norm-one elements $x, y$ such that $x y=y x=y$ and $x$ satisfies (2.3). Since $A \hookrightarrow B$ is a full embedding and $y \neq 0$, there are $b_{1}, \ldots, b_{m} \in B$ such that

$$
b_{1}^{*} y^{2} b_{1}+\ldots+b_{m}^{*} y^{2} b_{m}=1_{B} .
$$

This is proved in the same way as $[\mathbf{9}, 1.10]$.

Define $V \in \mathscr{L}\left(B, B^{m}\right)$ by arranging $y b_{1}, \ldots, y b_{m}$ into a column matrix. Then $V$ is an isometry because $V^{*} V=1_{B}$, and since $y$ acts as a unit on $x$, we have $\left(x \otimes 1_{m}\right) V=V$. This implies that

$$
\begin{aligned}
\| \varphi(a) 1_{B} & -V^{*}\left(a \otimes 1_{m}\right) V \| \\
& =\left\|\varphi(a) V^{*}\left(x^{2} \otimes 1_{m}\right) V-V^{*}\left(x \otimes 1_{m}\right)\left(a \otimes 1_{m}\right)\left(x \otimes 1_{m}\right) V\right\| \\
& \leqslant\left\|\varphi(a)\left(x^{2} \otimes 1_{m}\right)-x a x \otimes 1_{m}\right\|<\varepsilon
\end{aligned}
$$

for any $a \in \mathscr{F}$, using (2.3).

For $\varphi$ an arbitrary state, we argue as follows. By Krein-Milman theory we may approximate $\varphi$ by a convex combination of pure states, say

$$
\left\|\varphi(a)-\sum_{i=1}^{k} \lambda_{i} \varphi_{i}(a)\right\|<\varepsilon
$$

where $a \in \mathscr{F}$. Let $V_{i} \in \mathscr{L}\left(B, B^{m k}\right)$ be given by the first part of the proof, with

$$
V_{i}^{*} V_{i}=1_{B}, \quad V_{i} V_{i}^{*} \leqslant(0, \ldots, 0, \underbrace{1_{m} \otimes 1_{B}}_{i}, 0, \ldots, 0) .
$$

Then $V=\sum_{i=1}^{k} \sqrt{\lambda_{i}} V_{i}$ defines an isometry of $\mathscr{L}\left(B, B^{m k}\right)$, such that

$$
\begin{aligned}
\left\|\varphi(a)-V^{*}\left(a \otimes 1_{m k}\right) V\right\| & =\left\|\varphi(a)-\sum_{i=1}^{k} \lambda_{i} V_{i}^{*}\left(a \otimes 1_{m}\right) V_{i}\right\| \\
& \leqslant\left\|\varphi(a)-\sum_{i=1}^{k} \lambda_{i} \varphi_{i}(a)\right\|+\sum_{i=1}^{k} \lambda_{i}\left\|\varphi_{i}(a)-V_{i}^{*}\left(a \otimes 1_{m}\right) V_{i}\right\| \\
& <2 \varepsilon,
\end{aligned}
$$

where $a \in \mathscr{F}$.

The following generalizes a result of Lin [34].

THeOREM 2.22. Let $A$ be a unital separable $C^{*}$-algebra and let $\iota: A \hookrightarrow B$ be a unital full embedding. Then $d_{\iota}$ is nuclearly absorbing. 
Proof. Combine Lemma 2.21 and Proposition 2.19.

\section{K-theoretical preliminaries}

In this section we collect preliminary results and definitions behind our use of $K$ - and $K K$-theory.

\section{1. $K K$-theory}

In all of the paper, except in the proof of the lemma below, we are going to work exclusively with the Cuntz picture of the Kasparov groups $K K(A, B)$. We are only going to give a brief description of this realization here; more details are given in [14], but for complete information we refer the reader to the original sources $[\mathbf{1 1}, \mathbf{2 5}, \mathbf{5 1}]$, or to [28] for a detailed exposition.

The Cuntz picture is described in terms of pairs of representations

where

$$
(\varphi, \psi): A \rightarrow M(\mathscr{K}(H) \otimes B)
$$

$$
\varphi(a)-\psi(a) \in \mathscr{K}(H) \otimes B, \quad \text { for } a \in A .
$$

Such a pair will be called a Cuntz pair. They form a set denoted by $\mathbb{E}_{h}(A, B)$. A homotopy of Cuntz pairs consists of a Cuntz pair $(\Phi, \Psi): A \rightarrow M(\mathscr{K}(H) \otimes B[0,1])$, and the quotient of $\mathbb{E}_{h}(A, B)$ by homotopy equivalence becomes the Kasparov group $K K(A, B)$.

One defines groups $K K_{\text {nuc }}(A, B)$ by restricting attention to Cuntz pairs (and homotopies) consisting of strictly nuclear representations. Hence $K K=K K_{\text {nuc }}$ when $A$ or $B$ is nuclear. And one proves the following.

Lemma 3.1. Let $A$ be a unital separable $C^{*}$-algebra and let $B$ be a unital $C^{*}$-algebra. Let an admissible scalar representation $\theta$ be given. Then any $\alpha \in K K_{\mathrm{nuc}}(A, B)$ is represented by a strictly nuclear Cuntz pair of the form $(\rho, \widehat{\theta})$.

Proof. We use the fact that $K K_{\text {nuc }}(A, B)$ also has a Fredholm realization based on triples $\left(\varphi_{0}, \varphi_{1}, u\right)$ consisting of strictly nuclear $*$-homomorphisms $\varphi_{i}: A \rightarrow \mathscr{L}\left(E_{i}\right)$ intertwined up to compacts by $u \in \mathscr{L}\left(E_{0}, E_{1}\right)$ acting as a unitary, up to compacts, on the ranges of $\varphi_{i}$. In fact, according to [51, 2.6], $\alpha$ is represented by a triple of the form $(\widehat{\theta}, \widehat{\theta}, x)$. By using the standard simplification given by Proposition 17.4.3 of [3] we may assume that $x$ is a contraction. Finally we may replace $x$ by the unitary

$$
u=\left[\begin{array}{cc}
x & \left(1-x x^{*}\right)^{1 / 2} \\
-\left(1-x^{*} x\right)^{1 / 2} & x^{*}
\end{array}\right] .
$$

Hence, in $K K(A, B)$ we have $\alpha=[\widehat{\theta}, \widehat{\theta}, u]=\left[u \widehat{\theta} u^{*}, \widehat{\theta}, 1\right]$, and this translates in the Cuntz picture to the desired identity $\alpha=\left[u \widehat{\theta} u^{*}, \widehat{\theta}\right]$.

Note that any $*$-homomorphism $\varphi: A \rightarrow B$ induces an element $[\varphi] \in K K(A, B)$ via the Cuntz pair $(\varphi, 0)$. Similarly, any nuclear $*$-homomorphism $\varphi: A \rightarrow B$ induces an element $[\varphi] \in K K_{\mathrm{nuc}}(A, B)$. 


\subsection{K-theory}

When $B_{i}$ is a sequence of $\mathrm{C}^{*}$-algebras we denote by $\prod B_{i}$ their $\left(\ell_{\infty}\right.$-)product, and by $\sum B_{i}$ their $\left(c_{0^{-}}\right)$sum. It is well known, although perhaps not as well known as it should be, that the natural map $K_{*}\left(\prod B_{i}\right) \rightarrow \prod K_{*}\left(B_{i}\right)$ is not an isomorphism in general. In this section we are going to study, for large classes of $\mathrm{C}^{*}$-algebras $B_{i}$, injectivity properties of the natural map

$$
\underline{\eta}: \underline{\mathbf{K}}\left(\prod B_{i}\right) \rightarrow \prod \underline{\mathbf{K}}\left(B_{i}\right)
$$

where $\underline{\mathbf{K}}(-)$ denotes the sum of all $K$-theory groups with $\mathbb{Z} / n$ coefficients for all $n \geqslant 1$, defined by collecting the maps

$$
\eta^{i}: K_{*}\left(\prod B_{i}\right) \rightarrow \prod K_{*}\left(B_{i}\right), \quad \eta_{n}^{i}: K_{*}\left(\prod B_{i} ; \mathbb{Z} / n\right) \rightarrow \prod K_{*}\left(B_{i} ; \mathbb{Z} / n\right)
$$

induced by the projections $\pi_{i}$ : $\prod B_{i} \rightarrow B_{i}$. We also use this information to prove that surprisingly often, $\operatorname{Pext}\left(-, K_{*}\left(\prod B_{i} / \sum B_{i}\right)\right)$ will vanish.

When discussing maps from $\underline{\mathbf{K}}(A)$ to $\underline{\mathbf{K}}(B)$, we are only interested in collections of group homomorphisms which preserve the natural set of coefficient transformations and the Bockstein operations (see [49] and [18]). We denote the group of such maps by $\operatorname{Hom}_{\Lambda}(\underline{\mathbf{K}}(A), \underline{\mathbf{K}}(B))$.

If $B$ is a $C^{*}$-algebra we denote by $\operatorname{Proj}(B)$ the set of all self-adjoint projections in $B$. The $K$-theory class of a projection $p$ is denoted by $[p] \in K_{0}(B)$. If $B$ is unital, we let $\mathscr{U}_{n}(B)$ denote the unitary group of $\mathbf{M}_{n}(B)$.

$K$-theory of products. We are going to define quantities cco ('cancellation order'), pfo ('perforation order'), elo , elo $_{1}$ ('element lifting order') and ipo ('infinite height perturbation order') in $\mathbb{N} \cup\{\infty\}$ for any unital $C^{*}$-algebra $B$ by declaring

$$
\begin{aligned}
& \operatorname{cco}(B) \leqslant \ell \text { if whenever } p, q \in \operatorname{Proj}(B \otimes \mathscr{K}), \text { then }[p]=[q] \text { implies that } \\
& p \oplus \ell \cdot 1_{B} \sim q \oplus \ell \cdot 1_{B}, \\
& \operatorname{pfo}(B) \leqslant \ell \text { if for any } x \in K_{0}(B) \text { such that } n x \geqslant 0 \text { for some } n>0, \text { one has } \\
& x+\ell\left[1_{B}\right] \geqslant 0, \\
& \operatorname{elo}_{0}(B) \leqslant \ell \text { if the canonical map } \operatorname{Proj}\left(\mathbf{M}_{\ell}(B)\right) \rightarrow K_{0}(B) \text { is surjective, } \\
& \operatorname{elo}_{1}(B) \leqslant \ell \text { if the canonical map } \mathscr{U}_{\ell}(B) \rightarrow K_{1}(B) \text { is surjective, } \\
& \operatorname{ipo}(B) \leqslant \ell \text { if for any } x \text { in } K_{0}(B) \text { and any } n \neq 0, \text { there is } y \text { in } K_{0}(B) \text { such that } \\
&-\ell\left[1_{B}\right] \leqslant y \leqslant \ell\left[1_{B}\right] \text { and } x-y \in n K_{0}(B),
\end{aligned}
$$

and declaring the value to be $\infty$ when no such $\ell$ exists. For instance, elo $_{0}(B)=\infty$ if $K_{0}(B)_{+} \neq K_{0}(B)$. When $\left\{B_{i}\right\}_{i \in I}$ is a family of $\mathrm{C}^{*}$-algebras, we define $\operatorname{cco}\left(\left\{B_{i}\right\}\right)=\sup _{i} \operatorname{cco}\left(B_{i}\right)$, and so forth. We also write $\operatorname{rr}\left(\left\{B_{i}\right\}\right)=0$ when each $B_{i}$ has real rank 0 .

The following results - some of which are known, cf. [20] - demonstrate the relevance of these quantities to the map $\eta$. We denote the unit of $B_{i}$ by $1_{i}$ and the units of $\prod B_{i}$ and $\prod B_{i} / \sum B_{i}$ by $1_{\Pi}$ and $1_{\Pi / \Sigma}$, respectively. 
LEMma 3.2. Let $B_{i}$ be a sequence of unital $C^{*}$-algebras for which $\operatorname{cco}\left(\left\{B_{i}\right\}\right)<\infty$. Then $\eta^{0}$ is injective, and the image of $\eta^{0}$ equals

$$
\left\{\left(x_{i}\right) \in \prod K_{0}\left(B_{i}\right) \mid \exists M \in \mathbb{N} \forall i \in \mathbb{N}:-M\left[1_{i}\right] \leqslant x_{i} \leqslant M\left[1_{i}\right]\right\} .
$$

Furthermore, if $\eta^{0}(x)=\left(x_{i}\right)$ with each $x_{i} \geqslant 0$, then $x \geqslant 0$. If in addition pfo $\left(\left\{B_{i}\right\}\right)<\infty$, then $\operatorname{Im} \eta^{0}$ is a pure subgroup of $\prod K_{0}\left(B_{i}\right)$. If $\mathbf{e l o}_{0}\left(\left\{B_{i}\right\}\right)<\infty$, then $\eta^{0}$ is surjective.

Proof. Assume that $\operatorname{cco}\left(\left\{B_{i}\right\}\right) \leqslant \ell$. To prove injectivity, let

$$
x=\left[\left(p_{i}\right)\right]-\left[\left(q_{i}\right)\right] \in K_{0}\left(\prod B_{i}\right)
$$

be given by $p_{i}, q_{i} \in \mathbf{M}_{N}\left(B_{i}\right)$ and assume that $\eta^{0}(x)=0$. We have $p_{i} \oplus \ell \cdot 1_{i} \sim q_{i} \oplus \ell \cdot 1_{i}$, so that $\left(p_{i}\right) \oplus \ell \cdot 1_{\Pi} \sim\left(q_{i}\right) \oplus \ell \cdot 1_{\Pi}$, proving $x=0$. To prove that the image is contained in the set of bounded sequences, write $x \in K_{0}\left(\prod B_{i}\right)$ as $x=\left[\left(p_{i}\right)\right]-\left[\left(q_{i}\right)\right]$ with $p_{i}, q_{i} \in \mathbf{M}_{N}\left(B_{i}\right)$. By definition of positivity, $-N\left[1_{\Pi}\right] \leqslant x \leqslant N\left[1_{\Pi}\right]$ and we get the result by applying $\pi_{i}$. For the other inclusion, assume that $x_{i} \in K_{0}\left(B_{i}\right)$ is given with $-M\left[1_{i}\right] \leqslant x_{i} \leqslant M\left[1_{i}\right]$. We can write $x_{i}+M\left[1_{i}\right]=\left[r_{i}\right]$ for some projection $r_{i}$ in $B_{i} \otimes \mathscr{K}$, and since $\left[r_{i}\right] \leqslant 2 M\left[1_{i}\right]$, we get $2 M\left[1_{i}\right]=\left[r_{i} \oplus s_{i}\right]$ for some projection $s_{i}$ in $B_{i} \otimes \mathscr{K}$. Since $\operatorname{cco}\left(\left\{B_{i}\right\}\right) \leqslant \ell$, we see that $(2 M+\ell) \cdot 1_{i} \sim r_{i} \oplus s_{i} \oplus \ell \cdot 1_{i}$; hence there is $q_{i} \in \mathbf{M}_{2 M+\ell}\left(B_{i}\right)$ with $r_{i} \sim q_{i}$. Consequently, $x_{i}=\left[r_{i}\right]-M\left[1_{i}\right]$ can be represented as a difference of projections $\left[q_{i}\right]-M\left[1_{i}\right]$ where $q_{i} \in \mathbf{M}_{2 M+\ell}\left(B_{i}\right)$. Defining $q=\left(q_{i}\right) \in \mathbf{M}_{2 M+\ell}\left(\prod B_{i}\right)$, we find that $[q]-M\left[1_{\Pi}\right]$ is a preimage of $\left(x_{i}\right)$.

If $\eta^{0}(x)=\left(x_{i}\right)$ and every $x_{i}$ is positive, we have $0 \leqslant x_{i} \leqslant M\left[1_{i}\right]$ for some fixed $M$. Hence $x_{i}=\left[p_{i}\right]$ for some $p_{i}$ which we may assume lies in $M_{M+\ell}\left(B_{i}\right)$ as above. Consequently $p=\left(p_{i}\right)$ defines an element of $K_{0}\left(\prod B_{i}\right)$, and $x=[p]$ by injectivity of $\eta^{0}$. To establish purity when $\mathbf{p f o}\left(\left\{B_{i}\right\}\right) \leqslant \ell^{\prime}$, assume that $x=n y$ in $\prod K_{0}\left(B_{i}\right)$, where $x \in \operatorname{Im} \eta^{0}$, so that for some $M, M n\left[1_{i}\right] \pm x_{i} \geqslant 0$. We conclude that $\left(M+\ell^{\prime}\right)\left[1_{i}\right] \pm y_{i} \geqslant 0$, whence $y \in \operatorname{Im} \eta^{0}$. Proving surjectivity of $\eta^{0}$ when elo $_{0}\left(\left\{B_{i}\right\}\right)$ is finite is straightforward.

LEMmA 3.3. Let $B_{i}$ be a sequence of $C^{*}$-algebras. If $\mathbf{r r}\left(\left\{B_{i}\right\}\right)=0$, then $\eta^{1}$ is injective. If $\mathbf{e l o}_{1}\left(\left\{B_{i}\right\}\right)<\infty$, then $\eta^{1}$ is surjective.

Proof. To prove injectivity, we assume that $\eta^{1}(x)=0$ with $x=\left[\left(u_{i}\right)\right]$ and $u_{i} \in \mathbf{M}_{n}\left(B_{i}\right)$. By [33], $u_{i}$ is homotopic to $n \cdot 1_{i}$ within $\mathscr{U}_{n}\left(B_{i}\right)$, and because $\operatorname{rr}\left(B_{i}\right)=0$ the exponential lengths of $\mathbf{M}_{n}\left(B_{i}\right)$ are uniformly bounded (by $2 \pi$, see [32]), so we can choose short paths and combine them to one from $\left(u_{n}\right)$ to the unit of $\mathscr{U}_{n}\left(\prod B_{i}\right)$. Proving surjectivity of $\eta^{1}$ when $\operatorname{elo}_{1}\left(\left\{B_{i}\right\}\right)$ is finite is straightforward.

LEMma 3.4. Let $B_{i}$ be a sequence of unital $C^{*}$-algebras and abbreviate $\Pi=\prod B_{i}$ and $\Pi / \Sigma=\prod B_{i} / \sum B_{i}$. Then

(i) $\operatorname{cco}(\Pi), \operatorname{cco}(\Pi / \Sigma) \leqslant \operatorname{cco}\left(\left\{B_{i}\right\}\right)$,

(ii) $\mathbf{p f o}(\Pi)$, pfo $(\Pi / \Sigma) \leqslant \mathbf{p f o}\left(\left\{B_{i}\right\}\right)$ if $\operatorname{cco}\left(\left\{B_{i}\right\}\right)<\infty$,

(iii) elo $_{0}(\Pi)$, elo $_{0}(\Pi / \Sigma) \leqslant \operatorname{elo}_{0}\left(\left\{B_{i}\right\}\right)$ if $\operatorname{cco}\left(\left\{B_{i}\right\}\right)<\infty$,

(iv) $\mathbf{e l o}_{1}(\Pi), \operatorname{elo}_{1}(\Pi / \Sigma) \leqslant \operatorname{elo}_{1}\left(\left\{B_{i}\right\}\right)$ if $\mathbf{r r}\left(\left\{B_{i}\right\}\right)=0$,

(v) $\mathbf{i p o}(\Pi), \mathbf{i p o}(\Pi / \Sigma) \leqslant \mathbf{i p o}\left(\left\{B_{i}\right\}\right)$ if $\operatorname{cco}\left(\left\{B_{i}\right\}\right), \mathbf{p f o}\left(\left\{B_{i}\right\}\right)<\infty$. 
Proof. These claims all follow in a straightforward fashion from the properties of $\eta^{*}$ established in Lemmas 3.2 and 3.3. We only prove (v), which is the most involved result. Let $x \in K_{0}\left(\prod B_{i}\right)$ and $n$ be given and assume that ipo $\left(\left\{B_{i}\right\}\right) \leqslant \ell$. With $\eta^{0}(x)=\left(x_{i}\right)$, we can find $y_{i} \in K_{0}\left(B_{i}\right)$ with $-\ell\left[1_{i}\right] \leqslant y_{i} \leqslant \ell\left[1_{i}\right]$ and $x_{i}-y_{i} \in n K_{0}\left(B_{i}\right)$. We know from Lemma 3.2 that $\left(y_{i}\right)=\eta^{0}(y)$ for some $y \in K_{0}\left(\prod B_{i}\right)$ with $-\ell\left[1_{\Pi}\right] \leqslant y \leqslant \ell\left[1_{\Pi}\right]$. Since $\eta^{0}(x-y) \in n \prod K_{0}\left(B_{i}\right)$ by construction, we further conclude by purity that $\eta^{0}(x-y) \in n \operatorname{Im} \eta^{0}$ and, since $\eta^{0}$ is injective, that $x-y \in n K_{0}\left(\prod B_{i}\right)$.

To prove the result for $\Pi / \Sigma$, we note that

$$
0 \longrightarrow K_{*}(\Sigma) \longrightarrow K_{*}(\Pi) \longrightarrow K_{*}(\Pi / \Sigma) \longrightarrow 0
$$

is exact and apply the argument above to an $x \in K_{0}(\Pi)$ lifting the given element in $K_{0}(\Pi / \Sigma)$.

Algebraically compact $K$-groups. An abelian group $G$ is algebraically compact when $\operatorname{Pext}(-, G)$ vanishes. This class of groups is well studied (cf. [24, VII]), and we are going to use the characterization of it as those groups for which the following hold [26]:

(i) the subgroup $\bigcap_{n \in \mathbb{N}} n G$ is divisible;

(ii) $G$ is complete.

Here completeness refers to the $\mathbb{Z}$-adic topology (cf. [24, 7]), and completeness does not (as it does in [24]!) imply any separation properties.

Note that the quantities pfo and ipo make sense for general ordered abelian groups with order unit. We extend the notions to such groups and families of them in the obvious way. When $\left(G_{i}, 1_{i}\right)$ is a family of groups and order units, we define a 'bounded product' $\prod_{b}$ by

$$
\prod_{\mathrm{b}} G_{i}=\left\{\left(g_{i}\right) \mid \exists M \in \mathbb{N} \forall i \in I:-M 1_{i} \leqslant g_{i} \leqslant M 1_{i}\right\} .
$$

Lemma 3.5. Whenever $G_{i}$ is a sequence of abelian groups, then $\prod G_{i} / \sum G_{i}$ is algebraically compact. If, furthermore, all $G_{i}$ are ordered with order units, then $\prod_{\mathrm{b}} G_{i} / \sum G_{i}$ is algebraically compact provided that both $\mathbf{p f o}\left(\left\{G_{i}\right\}\right)$ and $\mathbf{i p o}\left(\left\{G_{i}\right\}\right)$ are finite.

Proof. When $X=\prod G_{i} / \sum G_{i}$, then $X$ is algebraically compact by [27]. When the $G_{i}$ are also ordered, let $X_{\mathrm{b}}=\prod_{\mathrm{b}} G_{i} / \sum G_{i}$ and consider $X_{\mathrm{b}}$ as a subgroup of $X$. We are going to prove that (i) and (ii) above hold for $X_{\mathrm{b}}$ from the fact that they hold for $X$.

Since pfo $\left(\left\{G_{i}\right\}\right)<\infty$, we immediately see that if $x \in X_{\mathrm{b}}$ and $x=m y$ in $X$, then $y \in X_{\mathrm{b}}$ if $m \neq 0$, by an argument very similar to the one at the end of the proof of Lemma 3.2. Note that this is stronger than mere purity of $X_{\mathrm{b}}$ as a subgroup of $X$. Fix $m \neq 0$ and $x \in \bigcap_{n \in \mathbb{N}} n X_{\mathrm{b}}$ and write $x=m y$ for $y \in \bigcap_{n \in \mathbb{N}} n X$. Applying this observation twice, we get

$$
y \in X_{\mathrm{b}} \cap \bigcap_{n \in \mathbb{N}} n X=\bigcap_{n \in \mathbb{N}}\left(n X \cap X_{\mathrm{b}}\right)=\bigcap_{n \in \mathbb{N}} n X_{\mathrm{b}}
$$

It remains to show that $X_{\mathrm{b}}$ is complete. To do so, we first note that for any given $x \in X$ there is $y \in X_{\mathrm{b}}$ such that $x-y \in \bigcap_{n \in \mathbb{N}} n X$. For when $\operatorname{ipo}\left(\left\{G_{i}\right\}\right) \leqslant \ell$ 
and $x=\left(x_{i}\right)+\sum G_{i}$ is given, we may find $y_{i}$ such that $-\ell 1_{i} \leqslant y_{i} \leqslant \ell 1_{i}$ and $x_{i}-y_{i} \in i ! G_{i}$. Clearly $y=\left(y_{i}\right)+\sum G_{i}$ has the desired property. Now let $\left(x_{n}\right)$ be Cauchy in $X_{\mathrm{b}}$, and recall that it converges to some $x \in X$. With $y$ chosen as above, $\left(x_{n}\right)$ also converges to $y \in X_{\mathrm{b}}$ in the $\mathbb{Z}$-adic topology of $X_{\mathrm{b}}$. This is because the $\mathbb{Z}$-adic topology of $X_{\mathrm{b}}$ coincides with the topology of $X_{\mathrm{b}}$ induced by the $\mathbb{Z}$-adic topology of $X$, since $X_{\mathrm{b}}$ is a pure subgroup of $X$.

COROLlary 3.6. Let $B_{i}$ be a sequence of unital $C^{*}$-algebras. Then

(i) $K_{1}\left(\prod B_{i} / \sum B_{i}\right)$ is algebraically compact if

$$
\boldsymbol{r r}\left(\left\{B_{i}\right\}\right)=0 \text { and } \operatorname{elo}_{1}\left(\left\{B_{i}\right\}\right)<\infty,
$$

(ii) $K_{0}\left(\prod B_{i} / \sum B_{i}\right)$ is algebraically compact if either

$$
\operatorname{cco}\left(\left\{B_{i}\right\}\right), \operatorname{elo}_{0}\left(\left\{B_{i}\right\}\right)<\infty \text { or } \operatorname{cco}\left(\left\{B_{i}\right\}\right), \operatorname{pfo}\left(\left\{B_{i}\right\}\right), \operatorname{ipo}\left(\left\{B_{i}\right\}\right)<\infty .
$$

Proof. Part (i) follows from Lemmas 3.3 and 3.5. Part (ii) follows from Lemmas 3.2 and 3.5.

\subsection{Partial maps on $\underline{\mathbf{K}}(-)$}

In this section we concern ourselves with associating $K$-theoretical data to completely positive contractions. Starting from such maps, say from $A$ to $B$, we shall be able to induce maps sending a finite set of projections representing a finite part of the $K$-theory of $A$ to elements of the $K$-theory of $B$.

Although there are advantages of doing this even for subsets representing elements of $K_{0}(A)$, the real strength of this approach only surfaces when we work with all of $\underline{\mathbf{K}}(A)$ and $\underline{\mathbf{K}}(B)$. Our partial maps do not descend to well-defined maps on subsets of $\underline{\mathbf{K}}(A)$, let alone to all of $\underline{\mathbf{K}}(A)$, but this fact does not cause any problems except notational and technical inconveniences.

As noted on page 661 of [15] we can realize any element of $\underline{\mathbf{K}}(-)$ as a difference of classes of projections from

$$
\underline{\operatorname{Proj}}(A)=\bigcup_{m \geqslant 1} \operatorname{Proj}\left(A \otimes C(\mathbb{T}) \otimes C\left(W_{m}\right) \otimes \mathscr{K}\right)
$$

where the $W_{m}$ are the Moore spaces of order $m$. This picture of $\underline{\mathbf{K}}(A)$ encompasses the standard pictures of $K_{0}(A)$ and $K_{*}(A)$ using projections of $A \otimes \mathscr{K}$ and $A \otimes C(\mathbb{T}) \otimes \mathscr{K}$, respectively, but not the standard picture of $K_{1}(A)$ using unitaries of $(A \otimes \mathscr{K})^{\sim}$. We need to pay special attention to this. Checking the facts stated as lemmas below is tedious but straightforward. We leave it to the reader with due apologies.

Definition 3.7. Let $A$ be a $\mathrm{C}^{*}$-algebra. A $\underline{\mathbf{K}}$-triple $(\mathscr{P}, \mathscr{G}, \delta)$ consists of finite subsets $\mathscr{P} \subseteq \operatorname{Proj}(A)$ and $\mathscr{G} \subseteq A$ and a $\delta>0$ chosen such that whenever $\varphi$ is a completely positive contraction which is $\delta$-multiplicative on $\mathscr{G}$, then

$$
\frac{1}{2} \notin \operatorname{sp}((\varphi \otimes \mathrm{id})(p))
$$

for each $p \in \mathscr{P}$, where id is the identity of $C(\mathbb{T}) \otimes C\left(W_{m}\right) \otimes \mathscr{K}$ for suitable $m$. A $K_{1}$-triple $(\mathscr{V}, \mathscr{G}, \delta)$ consists of finite subsets $\mathscr{V} \subseteq \mathscr{U}\left((A \otimes \mathscr{K})^{\sim}\right)$ and $\mathscr{G} \subseteq A$ and a $\delta>0$ chosen such that whenever $\varphi$ is a completely positive contraction which is $\delta$-multiplicative on $\mathscr{G}$, then

for each $v \in \mathscr{V}$.

$$
0 \notin \operatorname{sp}((\varphi \otimes \mathrm{id})(v))
$$


We define $K_{0^{-}}$and $K_{*}$-triples analogously to the $\underline{\mathbf{K}}$-triple case, by using projections in $A \otimes \mathscr{K}$ and $A \otimes C(\mathbb{T}) \otimes \mathscr{K}$, respectively.

The following lemma shows that any finite subset of projections or unitaries can be augmented to a triple of the appropriate kind.

Lemma 3.8. Let $A$ and $C$ be $C^{*}$-algebras, and fix finite sets $\mathscr{P}, \mathscr{V} \subseteq(A \otimes C)^{\sim}$ consisting of projections and unitaries, respectively. Then there exist $\delta>0$ and $a$ finite set $\mathscr{G} \subseteq A$ such that whenever $\varphi: A \rightarrow B$ is a unital completely positive map which is $\delta$-multiplicative on $\mathscr{G}$, then

$$
\frac{1}{2} \notin \mathrm{sp}\left(\left(\varphi \otimes \mathrm{id}_{C}\right)(p)\right), \quad 0 \notin \mathrm{sp}\left(\left(\varphi \otimes \operatorname{id}_{C}\right)(v)\right)
$$

for every $p \in \mathscr{P}$ and $v \in \mathscr{V}$.

Let $\chi_{0}:\left[0, \frac{1}{2}\right) \cup\left(\frac{1}{2}, 1\right] \rightarrow[0,1]$ be 0 on $\left[0, \frac{1}{2}\right)$ and 1 on $\left(\frac{1}{2}, 1\right]$, and let $\chi_{1}:(0,1] \rightarrow[0, \infty)$ be given by $\chi_{1}(x)=x^{-1 / 2}$.

Definition 3.9. Let $(\mathscr{P}, \mathscr{G}, \delta)$ be a $\mathbf{K}$-triple, and assume that $\varphi: A \rightarrow B$ is a completely positive contraction which is $\delta$-multiplicative on $\mathscr{G}$. We define $\varphi_{\sharp}: \mathscr{P} \rightarrow \underline{\mathbf{K}}(B)$ by

$$
\varphi_{\#}(p)=\left[\chi_{0}(\varphi \otimes \mathrm{id})(p)\right] .
$$

When $(\mathscr{V}, \mathscr{G}, \delta)$ is a $K_{1}$-triple, we define $\varphi_{\sharp}: \mathscr{V} \rightarrow K_{1}(B)$ by

$$
\varphi_{\#}(v)=\left[V \chi_{1}\left(V^{*} V\right)\right]
$$

where $V=(\varphi \otimes \mathrm{id})(v)$

Maps into $K_{0}(B)$ and $K_{*}(B)$ are defined from $K_{0}$-triples and $K_{*}$-triples similarly to the $\underline{\mathbf{K}}$-triple case.

Lemma 3.10. Let $A$ be a unital $C^{*}$-algebra and $(\mathscr{P}, \mathscr{G}, \delta)$ a $\underline{\mathbf{K}}$-triple. Let $\varphi: A \rightarrow B$ be a completely positive contraction which is $\delta$-multiplicative on $\mathscr{G}$ and let $j: B \rightarrow C$ be a unital $*$-homomorphism. Then $(j \varphi)_{\sharp}(p)=j_{*} \varphi_{\sharp}(p)$ for all $p \in \mathscr{P}$.

To establish the next two results, one may use the fact that the canonical map from $K_{0}(A)$ to $K_{1}(S A)$ is defined using scalar rotation matrices. The last result follows from the definitions.

Lemma 3.11. Whenever a $K_{*}$-triple $\left(\mathscr{P}_{*}, \mathscr{G}, \delta\right)$ is given, there exist a $K_{0}$-triple $\left(\mathscr{P}_{0}, \mathscr{G}_{0}, \delta_{0}\right)$ and a $K_{1}$-triple $\left(\mathscr{V}, \mathscr{G}_{1}, \delta_{1}\right)$ with $\delta_{i}<\delta$ and $\mathscr{G}_{i} \supseteq \mathscr{G}_{\text {such }}$ that if for two unital completely positive contractions $\varphi$ and $\psi$ which are $\delta_{i}$-multiplicative on $\mathscr{G}_{i}$,

$$
\varphi_{\sharp}(p)=\psi_{\sharp}(p) \text { and } \varphi_{\sharp}(v)=\psi_{\sharp}(v)
$$

for all $p \in \mathscr{P}_{0}$ and all $v \in \mathscr{V}$, then $\varphi_{\#}(p)=\psi_{\#}(p)$ for all $p \in \mathscr{P}_{*}$.

Lemma 3.12. Whenever a $K_{0}$-triple $\left(\mathscr{P}_{0}, \mathscr{G}_{0}, \delta_{0}\right)$ and a $K_{1}$-triple $\left(\mathscr{V}, \mathscr{G}_{1}, \delta_{1}\right)$ are given, there exists a $K_{*}$-triple $\left(\mathscr{P}_{*}, \mathscr{G}, \delta\right)$ with $\delta<\delta_{i}$ and $\mathscr{G} \supseteq \mathscr{G}_{i}$ such that if for two unital completely positive contractions $\varphi$ and $\psi$ which are $\delta$-multiplicative on $\mathscr{G}, \varphi_{\#}(p)=\psi_{\#}(p)$ for all $p \in \mathscr{P}_{*}$, then

$$
\varphi_{\#}(p)=\psi_{\#}(p) \quad \text { and } \quad \varphi_{\sharp}(v)=\psi_{\sharp}(v)
$$

for all $p \in \mathscr{P}_{0}$ and all $v \in \mathscr{V}$. 
Lemma 3.13. Let $(\mathscr{P}, \mathscr{G}, \delta)$ be a $\underline{\mathbf{K}}$-triple on A. There exist $\varepsilon>0$ and a finite set $\mathscr{F} \subseteq A$ such that if $\varphi$ and $\psi$ are completely positive contractions which are $\delta$-multiplicative on $\mathscr{G}$ and satisfy $\|\varphi(a)-\psi(a)\|<\varepsilon$ for all $a \in \mathscr{F}$, then $\varphi_{\#}(p)=\psi_{\#}(p)$ for all $p \in \mathscr{P}$.

\section{Uniqueness}

In the present section we present our uniqueness results as a sequence of successive refinements, at the cost of added assumptions.

We summarize our results in Table 1; the reader should be able to deduce the precise meaning of the various columns by comparing with the actual statements of the uniqueness results below. In the table we require tacitly that every $A$ is a separable $\mathrm{C}^{*}$-algebra and every map $\varphi, \psi$ is nuclear.

TABLE 1

\begin{tabular}{|c|c|c|c|c|c|}
\hline Ref. & $A$ & $B$ & $(A, B)$ & $\varphi, \psi$ & $\oplus$ \\
\hline 4.4 & & & $\begin{array}{l}\exists \gamma: A \rightarrow M(\mathscr{K} \otimes B) \\
\text { nuclearly absorbing }\end{array}$ & $\begin{array}{l}\text { morphisms } \\
{[\varphi]=[\psi]}\end{array}$ & $\gamma$ \\
\hline 4.5 & unital & unital & $\begin{array}{l}\exists \gamma: A \rightarrow M(\mathscr{K} \otimes B) \\
\text { unital } \\
\text { nuclearly absorbing } \\
\text { quasidiagonal }\end{array}$ & $\begin{array}{l}\text { morphisms } \\
{[\varphi]=[\psi]} \\
\varphi(1) \sim \psi(1)\end{array}$ & $\gamma_{n}$ \\
\hline 4.12 & $\begin{array}{l}\text { unital } \\
\text { simple } \\
\text { exact } \\
\text { UCT }\end{array}$ & $\begin{array}{l}\text { unital } \\
4.9\end{array}$ & $\begin{array}{l}\exists \iota: A \hookrightarrow B \\
\text { unital morphism }\end{array}$ & $\begin{array}{l}\text { morphisms } \\
\varphi_{*}=\psi_{*} \\
\varphi(1) \sim \psi(1)\end{array}$ & $n \cdot \iota$ \\
\hline 4.15 & $\begin{array}{l}\text { unital } \\
\text { simple } \\
\text { exact } \\
\text { UCT }\end{array}$ & $\begin{array}{l}\text { unital } \\
4.9\end{array}$ & $\begin{array}{l}\exists \tau: A \hookrightarrow B \\
\text { unital CP-map }\end{array}$ & $\begin{array}{l}\text { CP-maps } \\
\varphi_{\#}\left|\mathscr{P}=\psi_{\#}\right| \mathscr{P} \\
\varphi(1) \sim \psi(1)\end{array}$ & $n \cdot \tau$ \\
\hline
\end{tabular}

\subsection{Stable uniqueness}

We repeat the following definition and main result from [14].

Definition 4.1. If $\pi, \sigma: A \rightarrow \mathscr{L}(E)$ are representations, we say that $\pi$ and $\sigma$ are properly asymptotically unitarily equivalent and write $\pi \cong \sigma$ if there is a continuous path of unitaries $u:[0, \infty) \rightarrow \mathscr{U}\left(\mathscr{K}(E)+\mathbb{C} 1_{E}\right), u=\left(u_{t}\right)_{t \in[0, \infty)}$ such that for all $a \in A$,

(i) $\lim _{t \rightarrow \infty}\left\|u_{t} \pi(a) u_{t}^{*}-\sigma(a)\right\|=0$,

(ii) $u_{t} \pi(a) u_{t}^{*}-\sigma(a) \in \mathscr{K}(E)$, for all $t \in[0, \infty)$.

THeORem 4.2. Let $A$ be a (unital) separable $C^{*}$-algebra and let $B$ be a $\sigma$-unital $C^{*}$-algebra. Let $(\varphi, \psi): A \rightarrow M(\mathscr{K}(H) \otimes B)$ be a Cuntz pair consisting of (unital) strictly nuclear representations. Then the following are equivalent:

(i) $[\varphi, \psi]=0$ in $K K_{\text {nuc }}(A, B)$; 
(ii) there exists a (unital) strictly nuclear representation $\sigma: A \rightarrow M(\mathscr{K}(H) \otimes B)$ with $\varphi \oplus \sigma \approx \psi \oplus \sigma$;

(iii) for any (unital) nuclearly absorbing and strictly nuclear representation $\gamma: A \rightarrow M(\mathscr{K}(H) \otimes B), \varphi \oplus \gamma_{\infty} \approx \psi \oplus \gamma_{\infty}$.

It is proved in [14] that

$$
\varphi \oplus \sigma \approx \psi \oplus \sigma \Longleftrightarrow \varphi \oplus \gamma \approx \psi \oplus \gamma
$$

if $\sigma$ and $\gamma$ are asymptotically equivalent in a sense made precise in that paper. The same proof shows the following.

LEMMA 4.3. Let $\varphi, \psi, \gamma, \sigma: A \rightarrow M(\mathscr{K}(H) \otimes B)$ be representations. If $\varphi \oplus \gamma \approx \psi \oplus \gamma$, and $\gamma \sim \sigma$, then there exists a sequence of unitaries $v_{n} \in \mathscr{K}(H) \otimes B+\mathbb{C} 1$ such that

$$
\left\|v_{n}(\varphi \oplus \sigma)(a) v_{n}^{*}-(\psi \oplus \sigma)(a)\right\| \rightarrow 0 \quad \text { as } n \rightarrow \infty
$$

for any $a \in A$.

The previous lemma simplifies matters when working with uniqueness - it is necessary to work with asymptotic equivalence of cycles to characterize $K K$-theory as above, but to prove uniqueness results one only needs an approximate version of (i) $\Rightarrow$ (iii) above. Hence we get the following.

Corollary 4.4. Let $A$ be a separable $C^{*}$-algebra and let $B$ be a $\sigma$-unital $C^{*}$ algebra. Assume that $\varphi$ and $\psi$ are two nuclear $*$-homomorphisms from $A$ to $\mathscr{K}(H) \otimes B$ which satisfy $[\varphi]=[\psi]$ in $K K_{\text {nuc }}(A, B)$. Let $\gamma: A \rightarrow M(\mathscr{K}(H) \otimes B)$ be a nuclearly absorbing representation. Whenever a finite subset $\mathscr{F} \subseteq A$ and $\varepsilon>0$ are given, there exists a unitary $v \in \mathbf{M}_{3}(\mathscr{K}(H)) \otimes B+\mathbb{C} 1$ such that

$$
\left\|v\left[\begin{array}{ccc}
\varphi(a) & & \\
& 0 & \\
& & \gamma(a)
\end{array}\right] v^{*}-\left[\begin{array}{lll}
\psi(a) & & \\
& 0 & \\
& & \gamma(a)
\end{array}\right]\right\|<\varepsilon
$$

for all $a \in \mathscr{F}$.

Proof. The corollary follows from the non-unital version of Theorem 4.2 after the following remarks. Since $\varphi$ and $\psi$ assume only compact values, we have

$$
[\varphi, \psi]=[\varphi, 0]-[\psi, 0]=0
$$

in $K K_{\text {nuc }}(A, B)$. From Theorem 4.2, $\varphi \oplus \sigma \approx \psi \oplus \sigma$ for some strictly nuclear representation $\sigma: A \rightarrow M(\mathscr{K}(H) \otimes B)$. Now $\widehat{\gamma}=0 \oplus \gamma$ is (non-unital) nuclearly absorbing by Lemma 2.17, whether or not $\gamma$ is unital. Since $\varphi \oplus \sigma \oplus \widehat{\gamma} \approx \psi \oplus \sigma \oplus \widehat{\gamma}$ and $\sigma \oplus \widehat{\gamma} \sim \widehat{\gamma}$, we conclude that $v$ exists by Lemma 4.3 .

Achieving stable uniqueness. We are now going to employ the quasidiagonality condition of Definition 2.5 to truncate the absorbing representation in Corollary 4.4 to something more manageable in terms of classification.

We consider two $*$-homomorphisms $\varphi$ and $\psi$, between unital $\mathrm{C}^{*}$-algebras $A$ and $B$. To fix notation, let $\gamma: A \rightarrow M(\mathscr{K}(H) \otimes B)$ be a quasidiagonal unital representation. We consider a quasidiagonalization $\left(\gamma_{n}\right): A \rightarrow \mathbf{M}_{r_{n}}(B)$ by $\left(e_{n}\right)$ (see the paragraph 
following Definition 2.5), where we may and shall assume that $\left(e_{n}\right)$ has the additional property that

$$
e_{n} \varphi(a) e_{n}=\varphi(a) \text { and } e_{n} \psi(a) e_{n}=\psi(a),
$$

where $n \geqslant 1$.

TheOREm 4.5. Let $A$ be a unital, separable $C^{*}$-algebra and let $B$ be a unital $C^{*}$-algebra. Assume that there exists a unital quasidiagonal nuclearly absorbing representation $\gamma: A \rightarrow M(\mathscr{K}(H) B)$, and let $\left(\gamma_{n}\right): A \rightarrow \mathbf{M}_{r_{n}}(B)$ be a quasidiagonalization of $\gamma$ by $\left(e_{n}\right)$ as above.

Suppose that $\varphi, \psi: A \rightarrow B$ are two nuclear $*$-homomorphisms with $[\varphi]=[\psi]$ in $K K_{\text {nuc }}(A, B)$, such that $\varphi(1)$ is unitarily equivalent to $\psi(1)$. Then for any finite subset $\mathscr{F} \subseteq A$ and any $\varepsilon>0$, there exist an integer $n$ and a unitary $u \in \mathscr{U}_{r_{n}+1}(B)$ satisfying

$$
\left\|u\left[\begin{array}{cc}
\varphi(a) & \\
& \gamma_{n}(a)
\end{array}\right] u^{*}-\left[\begin{array}{ll}
\psi(a) & \\
& \gamma_{n}(a)
\end{array}\right]\right\|<\varepsilon
$$

for all $a \in \mathscr{F}$. Moreover we may arrange that $u\left(\varphi(1) \oplus \gamma_{n}(1)\right) u^{*}=\psi(1) \oplus \gamma_{n}(1)$.

Proof. After conjugating $\psi$ by a unitary in $B$ we may assume that $\varphi(1)=\psi(1)$. We are going to compress by $e_{n}^{\prime}=e_{n} \oplus e_{n} \oplus e_{n}$ (which is a quasicentral sequence in $\left.\mathbf{M}_{3}(\mathscr{K}(H)) \otimes B+\mathbb{C}_{3}\right)$, in the conclusion of Corollary 4.4. We have

$$
e_{n}^{\prime}\left[\begin{array}{ccc}
\varphi(a) & & \\
& 0 & \\
& & \gamma(a)
\end{array}\right] e_{n}^{\prime}=\left[\begin{array}{lll}
\varphi(a) & & \\
& 0 & \\
& & \gamma_{n}(a)
\end{array}\right]
$$

and a similar equation for $\psi$. It is crucial to our argument that the unitary $v$ provided by Corollary 4.4 satisfies $\left\|\left[v, e_{n}^{\prime}\right]\right\| \rightarrow 0$ because $v \in \mathbf{M}_{3}(\mathscr{K}(H)) \otimes B+\mathbb{C} 1_{3}$. Therefore by perturbing $e_{n}^{\prime} v e_{n}^{\prime}$ to a unitary $z$ within $\mathscr{U}_{3 r_{n}}(B)$, for some large $n$, we obtain

$$
\left\|z\left[\begin{array}{ccc}
\varphi(a) & & \\
& 0 & \\
& & \gamma_{n}(a)
\end{array}\right] z^{*}-\left[\begin{array}{lll}
\psi(a) & & \\
& 0 & \\
& & \gamma_{n}(a)
\end{array}\right]\right\|<\varepsilon
$$

for all $a \in \mathscr{F}$. Consider the projection $e=\varphi(1) \oplus \gamma_{n}(1)=\psi(1) \oplus \gamma_{n}(1)$. After a small perturbation of $z$ we may assume that $z e z^{*}=e$. Then $w=e z e$ is a partial isometry in $\mathbf{M}_{r_{n}+1}(B)$ with $w^{*} w=w w^{*}=e$, and the unitary

$$
u=w+1_{r_{n}+1}-e \in \mathscr{U}_{r_{n}+1}(B)
$$

will satisfy the conclusion of the theorem.

The following corollary is a good illustration of Theorem 4.5.

COROLlary 4.6. Let $A$ be a separable unital residually finite-dimensional $C^{*}$-algebra and let $B$ be a unital $C^{*}$-algebra. Let $\left(\pi_{n}\right)$ be a separating sequence of finite-dimensional representations of $A$ such that each $\pi_{n}$ is repeated infinitely many times. Define $\gamma_{n}=\pi_{1} \oplus \ldots \oplus \boldsymbol{\pi}_{n}$, with $\gamma_{n}: A \rightarrow \mathbf{M}_{r_{n}}(\mathbb{C}) \subseteq \mathbf{M}_{r_{n}}\left(\mathbb{C} 1_{B}\right)$. Let $\varphi, \psi: A \rightarrow B$ be two unital nuclear $*$-homomorphisms with $[\varphi]=[\psi]$ in $K K_{\text {nuc }}(A, B)$. Then there is a sequence of unitaries $u_{n} \in \mathscr{U}_{r_{n}+1}(B)$ such that

$$
\lim _{n \rightarrow \infty}\left\|u_{n}\left(\varphi(a) \oplus \gamma_{n}(a)\right) u_{n}^{*}-\psi(a) \oplus \gamma_{n}(a)\right\|=0,
$$

for $a \in A$. 
Proof. This follows from Theorem 4.5 with $\gamma=\pi_{1} \oplus \pi_{2} \oplus \ldots$. Note that since $\gamma$ is an admissible scalar representation, $\gamma$ is nuclearly absorbing by Proposition 2.18.

\subsection{Some classes of $C^{*}$-algebras}

We single out several classes of $\mathrm{C}^{*}$-algebras for easy reference.

Definition 4.7. We say that a separable $\mathrm{C}^{*}$-algebra $A$ satisfies the UCT if the diagram

$$
0 \longrightarrow \operatorname{Ext}\left(K_{*}(A), K_{*+1}(B)\right) \longrightarrow K K(A, B) \longrightarrow \operatorname{Hom}\left(K_{*}(A), K_{*}(B)\right) \longrightarrow 0
$$

is a short exact sequence for every $\sigma$-unital algebra $B$.

A large class of algebras satisfying the UCT was exhibited in [47]. If the separable $\mathrm{C}^{*}$-algebra $A$ satisfies the $\mathrm{UCT}$, then for any $\sigma$-unital $\mathrm{C}^{*}$-algebra $B$ the sequence

$$
0 \longrightarrow \operatorname{Pext}\left(K_{*}(A), K_{*+1}(B)\right) \longrightarrow K K(A, B) \longrightarrow \operatorname{Hom}_{\Lambda}(\underline{\mathbf{K}}(A), \underline{\mathbf{K}}(B)) \longrightarrow 0
$$

is also exact by [18].

REMARK 4.8. It is not known whether there exist separable nuclear $\mathrm{C}^{*}$-algebras not satisfying the UCT. If a separable $\mathrm{C}^{*}$-algebra $A$ satisfies the $\mathrm{UCT}$, then $A$ is $K K$-equivalent to a commutative $\mathrm{C}^{*}$-algebra; hence it is $K$-nuclear. In particular, $K K_{\text {nuc }}(A, B)=K K(A, B)$ by [51].

Definition 4.9. $\quad \mathrm{A} \mathrm{C}^{*}$-algebra $B$ is called an admissible target algebra if it is unital, has real rank 0 [7], and is such that

(i) whenever $p, q \in \operatorname{Proj}(B \otimes \mathscr{K})$, then $[p]=[q]$ implies $p \oplus 1_{B} \sim q \oplus 1_{B}$, and

(ii) the canonical map $\mathscr{U}_{1}(B) \rightarrow K_{1}(B)$ is surjective,

and if either

(iii. $\infty$ ) the canonical map $\operatorname{Proj}(B) \rightarrow K_{0}(B)$ is surjective or both of the following hold:

(iii.1) for any $x \in K_{0}(B)$ such that $n x \geqslant 0$ for some $n \geqslant 0$, one has $x+\left[1_{B}\right] \geqslant 0$,

(iv.1) for any $x \in K_{0}(B)$ and any $n \neq 0$, there is $y \in K_{0}(B)$ such that $-\left[1_{B}\right] \leqslant y \leqslant\left[1_{B}\right]$ and $x-y \in n K_{0}(B)$.

When needed, we distinguish between admissible target algebras satisfying (iii.1)-(iv.1) or (iii. $\infty$ ) by calling them admissible of finite type or infinite type, respectively. Examples will be given in Propositions 6.6 and 6.15 .

The point of this definition is that whenever a sequence of admissible targets is given, then both of the natural maps

$$
\begin{array}{r}
\operatorname{Hom}_{\Lambda}\left(\underline{\mathbf{K}}(A), \underline{\mathbf{K}}\left(\prod B_{i}\right)\right) \longrightarrow \prod \operatorname{Hom}_{\Lambda}\left(\underline{\mathbf{K}}(A), \underline{\mathbf{K}}\left(B_{i}\right)\right), \\
K K\left(A, \prod B_{i} / \sum B_{i}\right) \longrightarrow \operatorname{Hom}_{\Lambda}\left(\underline{\mathbf{K}}(A), \underline{\mathbf{K}}\left(\prod B_{i} / \sum B_{i}\right)\right)
\end{array}
$$

will be injective (the latter is in fact an isomorphism) for an $A$ satisfying the UCT. 
THEOREM 4.10. Let $B_{i}$ be a sequence of admissible target algebras of the same type and let $A$ be any $C^{*}$-algebra. Then:

(i) $\underline{\eta}: \underline{\mathbf{K}}\left(\prod B_{i}\right) \rightarrow \prod \underline{\mathbf{K}}\left(B_{i}\right)$ is injective;

(ii) the natural map $\operatorname{Hom}_{\Lambda}\left(\underline{\mathbf{K}}(A), \underline{\mathbf{K}}\left(\prod B_{i}\right)\right) \rightarrow \prod \operatorname{Hom}_{\Lambda}\left(\underline{\mathbf{K}}(A), \mathbf{K}\left(B_{i}\right)\right)$ is injective;

(iii) the natural map $K K\left(A, \prod B_{i} / \sum B_{i}\right) \rightarrow \operatorname{Hom}_{\Lambda}\left(\underline{\mathbf{K}}(A), \underline{\mathbf{K}}\left(\prod B_{i} / \sum B_{i}\right)\right)$ is an isomorphism if $A$ satisfies the UCT;

(iv) $\prod B_{i}$ and $\prod B_{i} / \sum B_{i}$ are admissible targets.

Proof. For (i), decompose $\eta$ into maps $\eta^{*}$ and $\eta_{n}^{*}$. We find from Lemmas 3.2 and 3.3 that the $\eta^{*}$ are injections whose images are pure subgroups. Injectivity of $\eta_{n}^{*}$ then follows by a diagram chase on

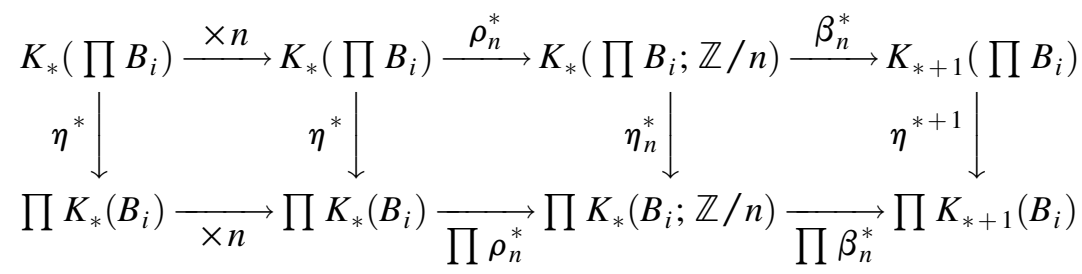

Claim (ii) is a direct consequence of (i), and (iii) follows by combining the UMCT (4.1) with Corollary 3.6. Finally, (iv) follows by Lemma 3.4.

Remark 4.11. As is clear from $\S 3.2$, we can get injectivity for the maps discussed above by asserting considerably weaker versions of (iii)-(iv). On the other hand, $B_{i}=C([0,1])$ is a counterexample to injectivity in (4.2) and $B_{i}=\mathbb{C}$ is a counterexample to injectivity in (4.3).

\subsection{Stable uniqueness with bounds}

Let $A$ be a separable unital $\mathrm{C}^{*}$-algebra and let $\iota: A \rightarrow B$ be a unital full embedding. If one specializes to the case $\gamma=d_{\iota}$ in Theorem 4.5, one obtains a stable approximate unitary equivalence of the form

$$
\left\|u\left[\begin{array}{cc}
\varphi(a) & \\
& n \cdot \iota(a)
\end{array}\right] u^{*}-\left[\begin{array}{ll}
\psi(a) & \\
& n \cdot \iota(a)
\end{array}\right]\right\|<\varepsilon .
$$

To make such a result useful in our quest to classify $\mathrm{C}^{*}$-algebras, we need to refine our uniqueness results to the effect of controlling the number $n$. More specifically, we need to know that these integers can be chosen uniformly with respect to the targets; that is, only depending on the source algebra and, of course, the requirements on how closely the two morphisms are to agree after composition by the unitary.

We also need to strengthen the theorem to allow for maps which are not *-homomorphisms and only induce the same element locally in $\operatorname{Hom}_{\Lambda}(\underline{\mathbf{K}}(A), \underline{\mathbf{K}}(B))$, rather than in $K K(A, B)$. To achieve such results, we are going to work with products of $\mathrm{C}^{*}$-algebras, and we are going to depend on the results in $\S 3.2$ regarding their $K$-theory. If $A$ satisfies the UCT, then it follows from (4.1) that $\operatorname{Hom}_{\Lambda}(\underline{\mathbf{K}}(A), \underline{\mathbf{K}}(B))$ is isomorphic to Rørdam's group $K L(A, B)[\mathbf{4 6}]$. 


\section{Bounded stable uniqueness for *-homomorphisms}

THEOREM 4.12. Let $A$ be a simple, unital, exact, separable $C^{*}$-algebra satisfying the UCT. Then for any finite subset $\mathscr{F} \subseteq A$ and any $\varepsilon>0$, there exists $n \in \mathbb{N}$ with the following property. For any admissible target $B$, any unital embedding $\iota: A \rightarrow B$ and any pair of nuclear $*$-homomorphisms $\varphi, \psi: A \rightarrow B$ such that $\varphi_{*}=\psi_{*}$ in $\operatorname{Hom}_{\Lambda}(\underline{\mathbf{K}}(A), \underline{\mathbf{K}}(B))$, and $\varphi(1)$ is unitarily equivalent to $\psi(1)$, there exists a unitary $u \in \mathscr{U}_{n+1}(B)$ such that

$$
\left\|u\left[\begin{array}{ll}
\varphi(a) & \\
& n \cdot \iota(a)
\end{array}\right] u^{*}-\left[\begin{array}{ll}
\psi(a) & \\
& n \cdot \iota(a)
\end{array}\right]\right\|<\varepsilon,
$$

for all $a \in \mathscr{F}$. Moreover we may arrange that $u(\varphi(1) \oplus n \cdot 1) u^{*}=\psi(1) \oplus n \cdot 1$.

Proof. We have $K K_{\text {nuc }}(A, B)=K K(A, B)$ by Remark 4.8. Seeking a contradiction, fix $\mathscr{F}$ and $\varepsilon$ for which the theorem fails. Then for any $i$ we choose an admissible target algebra $B_{i}$ equipped with an embedding $\iota_{i}: A \rightarrow B_{i}$, and $\varphi_{i}$ and $\psi_{i}$ nuclear $*$-homomorphisms with $\varphi_{i *}=\psi_{i *}$, and $\varphi_{i}(1)$ unitarily equivalent to $\psi_{i}(1)$, yet

$$
\inf _{u \in \mathscr{U}_{i+1}(B)} \max _{a \in \mathscr{F}}\left\|u\left[\begin{array}{ll}
\varphi_{i}(a) & \\
& i \cdot \iota_{i}(a)
\end{array}\right] u^{*}-\left[\begin{array}{ll}
\psi_{i}(a) & \\
& i \cdot \iota_{i}(a)
\end{array}\right]\right\| \geqslant \varepsilon .
$$

We define $\Phi, \Psi, I: A \rightarrow \prod B_{i}$ in the obvious way. The homomorphisms $\Phi$ and $\Psi$ are nuclear since $\varphi_{i}$ and $\psi_{i}$ are nuclear and $A$ is exact (see [12, 3.3]). By composing with the canonical map we get $\dot{\Phi}, \dot{\Psi}, \dot{I}: A \rightarrow \prod B_{i} / \sum B_{i}$, with $\dot{\Phi}$ and $\dot{\Psi}$ nuclear. Since $\Phi$ and $\Psi$ induce the same element of $\prod \operatorname{Hom}_{\Lambda}\left(\underline{\mathbf{K}}(A), \underline{\mathbf{K}}\left(B_{i}\right)\right)$ by construction, we get, from Theorem 4.10(ii), $\Phi_{*}=\Psi_{*}$ in $\operatorname{Hom}_{\Lambda}\left(\underline{\mathbf{K}}(A), \underline{\mathbf{K}}\left(\prod B_{i}\right)\right)$. Then of course $(\dot{\Phi})_{*}=(\dot{\Psi})_{*}$ also, and by Theorem 4.10(iii) we get $[\dot{\Phi}]_{K K}=[\dot{\Psi}]_{K K}$ in $K K\left(A, \prod B_{i} / \sum B_{i}\right)$.

Since $\dot{I}: A \rightarrow \prod B_{i} / \sum B_{i}$ is a unital full embedding, we conclude by Theorem 4.5 that there exist $n$ and a unitary $w \in \mathscr{U}_{n+1}\left(\prod B_{i} / \sum B_{i}\right)$ intertwining $\dot{\Phi} \oplus n \cdot \dot{I}$ and $\dot{\Psi} \oplus n \cdot \dot{I}$ up to $\varepsilon$ on $\mathscr{F}$. Let $u=\left(u_{i}\right) \in \mathscr{U}_{n+1}\left(\prod B_{i}\right)$ be a unitary lifting $w$. Then

$$
\lim \sup _{i} \max _{a \in \mathscr{F}}\left\|u_{i}\left[\begin{array}{cc}
\varphi_{i}(a) & \\
& n \cdot \iota_{i}(a)
\end{array}\right] u_{i}^{*}-\left[\begin{array}{ll}
\psi_{i}(a) & \\
& n \cdot \iota_{i}(a)
\end{array}\right]\right\|<\varepsilon,
$$

yielding a contradiction after projecting onto $\mathbf{M}_{n+1}\left(B_{i}\right)$ for large $i$. The last part of the proof is done exactly as the last part of the proof of Theorem 4.5.

REMARK 4.13. If $\varphi$ and $\psi$ are as in the conclusion of either Theorem 4.5 or Theorem 4.12, it follows immediately from the cancellative nature of $K$-theory that $\varphi_{*}=\psi_{*}: \underline{\mathbf{K}}(A) \rightarrow \underline{\mathbf{K}}(B)$.

REMARK 4.14. Under assumptions restricting the algebraic complexity on $K_{*}(A)$ and $K_{*}(B)$ the result above can be simplified somewhat. If we add, for instance, the assumptions that $K_{0}(A)$ be torsion free and $K_{0}(B)$ be divisible, we need only require that $\varphi_{*}=\psi_{*}$ on $K_{*}(A)$. This is done by basing the proof instead on injectivity of the maps

$$
\begin{gathered}
\operatorname{Hom}\left(K_{*}(A), K_{*}\left(\prod B_{i}\right)\right) \rightarrow \prod \operatorname{Hom}\left(K_{*}(A), K_{*}\left(B_{i}\right)\right), \\
K K\left(A, \prod B_{i} / \sum B_{i}\right) \rightarrow \operatorname{Hom}\left(K_{*}(A), K_{*}\left(\prod B_{i} / \sum B_{i}\right)\right) .
\end{gathered}
$$


We get the latter by applying the UCT and noting that since $K_{0}(A)$ is torsion free,

$$
\operatorname{Ext}\left(K_{0}(A), K_{1}\left(\prod B_{i} / \sum B_{i}\right)\right)=\operatorname{Pext}\left(K_{0}(A), K_{1}\left(\prod B_{i} / \sum B_{i}\right)\right)=0
$$

from Corollary 3.6(i), and that

$$
\operatorname{Ext}\left(K_{1}(A), K_{0}\left(\prod B_{i} / \sum B_{i}\right)\right)=0
$$

since, along the lines of the first half of Lemma 3.5, if all $K_{0}\left(B_{i}\right)$ are divisible, then so is $K_{0}\left(\prod B_{i}\right)$.

Bounded stable uniqueness for approximate morphisms. We refer the reader to $\S 3.3$ for a discussion of partially defined maps on $\underline{\mathbf{K}}(-)$ and a definition of $\underline{\mathbf{K}}$-triples.

Our next result was inspired by the main result in the version of [34] that we had received from the author when we began our work in February 1998. The original statement is corrected and generalized below. A significant difficulty in the proof below comes from handling the $K$-theory for products of $\mathrm{C}^{*}$-algebras; a point which was overlooked in that version. Our methods for overcoming this difficulty were developed in $\S 3.2$, leading us to Theorem 4.10 .

THEOREM 4.15. Let $A$ be a simple, unital, exact, separable $C^{*}$-algebra satisfying the UCT. For any finite subset $\mathscr{F} \subseteq A$ and any $\varepsilon>0$, there exist $n \in \mathbb{N}$, and a $\mathbf{K}$-triple $(\mathscr{P}, \mathscr{G}, \delta)$ with the following property. For any admissible target $B$, and any three completely positive contractions $\varphi, \psi, \tau: A \rightarrow B$ which are $\delta$-multiplicative on $\mathscr{G}$, with $\tau$ unital, $\varphi$ and $\psi$ nuclear, and $\varphi_{\#}(p)=\psi_{\sharp}(p)$ in $\underline{\mathbf{K}}(B)$ for all $p \in \mathscr{P}$, and such that $\varphi(1)$ and $\psi(1)$ are unitarily equivalent projections, there exists a unitary $u \in \mathscr{U}_{n+1}(B)$ such that

$$
\left\|u\left[\begin{array}{cc}
\varphi(a) & \\
& n \cdot \tau(a)
\end{array}\right] u^{*}-\left[\begin{array}{ll}
\psi(a) & \\
& n \cdot \tau(a)
\end{array}\right]\right\|<\varepsilon
$$

for all $a \in \mathscr{F}$. One may arrange that $u(\varphi(1) \oplus n \cdot 1) u^{*}=\psi(1) \oplus n \cdot 1$.

Proof. We have $K K_{\text {nuc }}(A, B)=K K(A, B)$ by Remark 4.8. Seeking a contradiction we suppose that there are $\mathscr{F}$ and $\varepsilon$ such that with $n=n(A, \mathscr{F}, \varepsilon)$ provided by Theorem 4.12, no K-triple will work. We choose sequences of $\underline{\mathbf{K}}$-triples $\left(\mathscr{P}_{i}, \mathscr{G}_{i}, \delta_{i}\right)$ with the following properties:

(i) $\mathscr{P}_{i} \subseteq \mathscr{P}_{i+1}$, and $\bigcup_{i \in \mathbb{N}} \mathscr{P}_{i}$ exhausts the semigroup

$$
\bigcup_{m \in \mathbb{N}} \operatorname{Proj}\left(A \otimes C(\mathbb{T}) \otimes C\left(W_{m}\right) \otimes \mathscr{K}\right) / \approx ;
$$

(ii) $\mathscr{G}_{i} \subseteq \mathscr{G}_{i+1}$ and $\overline{\bigcup_{i} \mathscr{G}_{i}}=A$;

(iii) $\delta_{i}>\delta_{i+1}$ and $\delta_{i} \rightarrow 0$.

By our assumption, we can then choose admissible targets $B_{i}$, which we may assume are of the same type, and $\varphi_{i}, \psi_{i}$ and $\tau_{i}$ which are $\delta_{i}$-multiplicative on $\mathscr{G}_{i}$, with $\varphi_{i}$ and $\psi_{i}$ nuclear, and satisfy $\left(\varphi_{i}\right)_{\sharp}(p)=\left(\psi_{i}\right)_{\sharp}(p)$ in $\underline{\mathbf{K}}(B)$ for all $p \in \mathscr{P}_{i}$, and $\varphi_{i}(1)$ and $\psi_{i}(1)$ are unitarily equivalent projections; yet

$$
\inf _{u \in \mathscr{U}_{n+1}(B)} \max _{a \in \mathscr{F}}\left\|u\left[\begin{array}{ll}
\varphi_{i}(a) & \\
& n \cdot \tau_{i}(a)
\end{array}\right] u^{*}-\left[\begin{array}{cc}
\psi_{i}(a) & \\
& n \cdot \tau_{i}(a)
\end{array}\right]\right\| \geqslant \varepsilon .
$$


Define $\Phi, \Psi, T: A \rightarrow \prod B_{i}$ from the sequences $\left(\varphi_{i}\right),\left(\psi_{i}\right)$ and $\left(\tau_{i}\right)$, and compose with the canonical map to get $\dot{\Phi}, \dot{\Psi}, \dot{T}: A \rightarrow \prod B_{i} / \sum B_{i}$. These maps are in fact *-homomorphisms by (ii) and (iii) above, so $\dot{T}$ provides a unital full embedding of $A$ into $\prod B_{i} / \sum B_{i}$, and $\dot{\Phi}$ and $\dot{\Psi}$ induce maps

$$
\dot{\Phi}_{*}, \dot{\Psi}_{*}: \underline{\mathbf{K}}(A) \rightarrow \underline{\mathbf{K}}\left(\prod B_{i} / \sum B_{i}\right) .
$$

We are going to show that $\dot{\Phi}_{*}=\dot{\Psi}_{*}$.

We may check this on $p \in \mathscr{P}_{j}$ by (i) above. Let $C=C(\mathbb{T}) \otimes C\left(W_{m}\right) \otimes \mathscr{K}$, with $m$ chosen appropriately. In the diagram

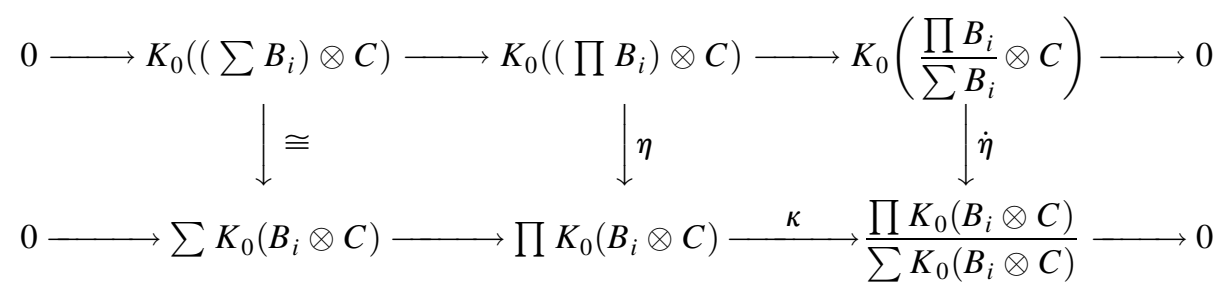

$\eta$ is injective from Theorem 4.10(i) because it can be naturally identified with a component of $\boldsymbol{\eta}$. It is not hard to check that the above diagram has exact rows. (The first row is induced by a quasidiagonal extension.) By the five-lemma, $\dot{\eta}$ is also injective, and hence it suffices to show that

$$
\dot{\eta}\left[\left(\dot{\Phi} \otimes \mathrm{id}_{C}\right)(p)\right]=\dot{\eta}\left[\left(\dot{\Psi} \otimes \operatorname{id}_{C}\right)(p)\right] .
$$

With $\chi_{0}$ as in $\S 3.3$, lift $(\dot{\Phi} \otimes$ id $)(p)$ first to a self-adjoint element $(\Phi \otimes$ id $)(p)$ in $\left(\prod B_{i}\right) \otimes C$, and then to a projection $q$ in

$$
\chi_{0}((\Phi \otimes \mathrm{id})(p))+\left(\sum B_{i}\right) \otimes C \subseteq\left(\prod B_{i}\right) \otimes C .
$$

Then $\eta([q])=\left(\left[q_{i}\right]\right)$ where $q_{i}=\chi_{0}\left(\left(\varphi_{i} \otimes \mathrm{id}\right)(p)\right)$ for all $i$ larger than some $i_{\Phi}$, since $\eta$ is induced by a family of $*$-homomorphisms. We conclude that

$$
\dot{\eta}\left((\dot{\Phi} \otimes \mathrm{id})_{*}(p)\right)=\kappa \eta([q])=\left[\chi_{0}\left(\left(\varphi_{i} \otimes \mathrm{id}\right)(p)\right)\right]_{i \geqslant i_{\Phi}}+\sum K_{0}\left(B_{i} \otimes C\right) .
$$

Similarly,

$$
\dot{\eta}\left((\dot{\Psi} \otimes \mathrm{id})_{*}(p)\right)=\left[\chi_{0}\left(\left(\psi_{i} \otimes \mathrm{id}\right)(p)\right)\right]_{i \geqslant i_{\Psi}}+\sum K_{0}\left(B_{i} \otimes C\right),
$$

and these elements agree since the sequences coincide for $i \geqslant i_{\Phi}, i_{\Psi}, j$.

Note that $\Phi$ and $\Psi$, and hence also $\dot{\Phi}$ and $\dot{\Psi}$, are nuclear since $\varphi_{i}$ and $\psi_{i}$ are nuclear and $A$ is exact [12]. Having proved that $\dot{\Phi}_{*}=\dot{\Psi}_{*}$, and since $\dot{\Phi}(1)$ is unitarily equivalent to $\dot{\Psi}(1)$, we may thus apply Theorem 4.12 to find a unitary $w \in \mathscr{U}_{n+1}\left(\prod B_{i} / \sum B_{i}\right)$ such that

$$
\left\|w\left[\begin{array}{cc}
\dot{\Phi}(a) & \\
& n \cdot \dot{T}(a)
\end{array}\right] w^{*}-\left[\begin{array}{ll}
\dot{\Psi}(a) & \\
& n \cdot \dot{T}(a)
\end{array}\right]\right\|<\varepsilon,
$$

for all $a \in \mathscr{F}$. Note that we may apply Theorem 4.12 since whenever $B_{i}$ is a sequence of admissible targets, $\prod B_{i} / \sum B_{i}$ is an admissible target by Theorem 4.10(iv). We finish the argument by lifting the unitary as above. 
REMARK 4.16. As in Remark 4.14, the premises of Theorem 4.15 simplify under extra assumptions on $K_{*}(A)$ and $K_{*}(B)$. If $K_{0}(A)$ is torsion free and $K_{0}(B)$ is divisible, one needs only check that $\varphi$ and $\psi$ agree on a $K_{*}$-triple. This is because divisibility passes from $K_{0}\left(B_{i}\right)$ to $K_{0}\left(\prod B_{i} / \sum B_{i}\right)$ as outlined in Remark 4.14.

\section{Existence}

In this section we show how to realize locally a given $K K$-element by a difference of completely positive contractions (see Theorem 5.5).

\subsection{Realizing group homomorphisms}

We refer the reader to $\S 3.3$ for the definition of $\mathbf{K}$-triples. Let $\chi_{0}$ be the characteristic function of $\left(\frac{1}{2}, 1\right]$.

Lemma 5.1. Let $D$ be a unital $C^{*}$-algebra. Let $p \in D$ be a projection and let $x \in D$ be a positive contraction. Then:

(i) $\left|x-\frac{1}{2}\right| \geqslant\left(\frac{1}{2}-\|x-p\|\right) 1$;

(ii) $\operatorname{sp}(x) \subset[0, \lambda] \cup[1-\lambda, 1]$, where $\lambda$ is the smaller root of

$$
\lambda^{2}-\lambda+\left\|x^{2}-x\right\|=0 ;
$$

thus if $\left\|x^{2}-x\right\|<\frac{1}{4}$, then $\chi_{0}(x) \in D$ is a projection with $\left\|x-\chi_{0}(x)\right\| \leqslant \lambda$;

(iii) if $\left(f_{n}\right)$ is a sequence of projections such that $\left[p, f_{n}\right] \rightarrow 0$ as $n \rightarrow \infty$, then there is a sequence of unitaries $\left(v_{n}\right)$ in $D$, such that $\left[v_{n} p v_{n}^{*}, f_{n}\right]=0$, for $n \geqslant 1$, and $\left\|v_{n}-1\right\| \rightarrow 0$ as $n \rightarrow \infty$.

Proof. For (i), we may assume that $D$ acts on a Hilbert space $H$, and for (ii), that $D=C(X)$ with $X$ compact. After these assumptions the claims become elementary. In (iii), we may assume that $\left\|\left[p, f_{n}\right]\right\|<\frac{1}{4}$ for all $n$. Define $q_{n}=\chi_{0}\left(p f_{n} p\right)+\chi_{0}\left(p^{\perp} f_{n} p^{\perp}\right)$. Then $\left[q_{n}, p\right]=0$, for $n \geqslant 1$, and $\left\|q_{n}-f_{n}\right\| \rightarrow 0$ as $n \rightarrow \infty$. By functional calculus, there is a sequence of unitaries $\left(v_{n}\right)$ in $D$ such that $q_{n}=v_{n}^{*} f_{n} v_{n}$ and $\left\|v_{n}-1\right\| \rightarrow 0$ as $n \rightarrow \infty$. The sequence $\left(v_{n}\right)$ has the desired properties.

Lemma 5.2. Let $E_{i}$ and $F_{i}$ be projections in $M(\mathscr{K}(H) \otimes B)$ with

$$
\left\|E_{0}-E_{1}\right\|<\frac{1}{2}, \quad\left\|F_{0}-F_{1}\right\|<\frac{1}{2}, \quad E_{i}-F_{i} \in \mathscr{K}(H) \otimes B
$$

for $i \in\{0,1\}$. Then $\left[E_{0}, F_{0}\right]=\left[E_{1}, F_{1}\right]$ in $K K(\mathbb{C}, B)$.

Proof. If $X_{t}=(1-t) E_{0}+t E_{1}$ and $Y_{t}=(1-t) F_{0}+t F_{1}$, then

$$
\operatorname{sp}\left(X_{t}\right), \operatorname{sp}\left(Y_{t}\right) \subseteq\left[0, \frac{1}{2}\right) \cup\left(\frac{1}{2}, 1\right]
$$

for all $t \in[0,1]$, by Lemma 5.1(i). Define $E_{t}=\chi_{0}\left(X_{t}\right)$ and $F_{t}=\chi_{0}\left(Y_{t}\right)$. Then $\left(E_{t}, F_{t}\right)$, for $0 \leqslant t \leqslant 1$, is a homotopy of Cuntz pairs from $\left(E_{0}, F_{0}\right)$ to $\left(E_{1}, F_{1}\right)$.

LemMa 5.3. If $E$ and $F$ are projections in $M(\mathscr{K}(H) \otimes B)$ with $E-F \in \mathscr{K}(H) \otimes B$ (that is, $(E, F)$ is a Cuntz pair), and $e$ is a projection in 
$\mathscr{K}(H) \otimes B$ with

$$
\|[e, E]\|<\frac{1}{9}, \quad\|[e, F]\|<\frac{1}{9}, \quad\left\|e^{\perp}(E-F) e^{\perp}\right\|<\frac{1}{9},
$$

then the natural isomorphism between $K K(\mathbb{C}, B)$ and $K_{0}(B)$ takes $[E, F]$ to

$$
\left[\chi_{0}(e E e)\right]-\left[\chi_{0}(e F e)\right]
$$

Proof. Note that

$$
\left\|(e E e)^{2}-e E e\right\| \leqslant\|[e, E]\| \leqslant \frac{1}{9} .
$$

Therefore $\left\|e E e-\chi_{0}(e E e)\right\| \leqslant \lambda$ with $\lambda=\frac{1}{6}(3-\sqrt{5})$ by Lemma 5.1(ii). We have similar estimates for $e^{\perp} E e^{\perp}, e F e$ and $e^{\perp} F e^{\perp}$.

Let $g=\chi_{0}(e E e), g^{\prime}=\chi_{0}\left(e^{\perp} E e^{\perp}\right), h=\chi_{0}(e F e)$, and $h^{\prime}=\chi_{0}\left(e^{\perp} F e^{\perp}\right)$. Then

$$
\begin{aligned}
\left\|E-\left(g+g^{\prime}\right)\right\| \leqslant & \left\|E-e E e-e^{\perp} E e^{\perp}\right\| \\
& +\left\|e E e-\chi_{0}(e E e)\right\|+\left\|e^{\perp} E e^{\perp}-\chi_{0}\left(e^{\perp} E e^{\perp}\right)\right\| \\
\leqslant & \frac{2}{9}+2 \lambda<\frac{1}{2} .
\end{aligned}
$$

Similarly, one shows that

$$
\left\|F-\left(h+h^{\prime}\right)\right\| \leqslant \frac{2}{9}+2 \lambda<\frac{1}{2}, \quad\left\|g^{\prime}-h^{\prime}\right\| \leqslant \frac{2}{9}+\lambda<\frac{1}{2},
$$

and all those quantities are compact. Moreover $g, g^{\prime}, h$ and $h^{\prime}$ are all projections and we may apply Lemma 5.2 to get

$$
[E, F]=\left[g+g^{\prime}, h+h^{\prime}\right]=[g, h]+\left[g^{\prime}, h^{\prime}\right]=[g, h]+\left[g^{\prime}, g^{\prime}\right]=[g, h]
$$

in $K K(\mathbb{C}, B)$. Since $g, h \in \mathscr{K}(H) \otimes B$, the isomorphism between $K K(\mathbb{C}, B)$ and $K_{0}(B)$ takes $[g, h]$ to $[g]-[h]$.

We now come to the main results in this section. The reader is referred to $\S 2.2$ for examples of quasidiagonal strictly nuclear nuclearly absorbing representations.

Any element $\alpha \in K K(A, B)$ induces a morphism $\alpha_{*}: \underline{\mathbf{K}}(A) \rightarrow \underline{\mathbf{K}}(B)$. Let us first describe the map induced by $\alpha$ on $K_{0}$. If $A$ is unital and if $p$ is a projection in $\mathbf{M}_{n}(A)$, and $\alpha$ is given by a Cuntz pair $(\tau, \gamma)$, then $\alpha_{*}$ takes $[p] \in K_{0}(A) \cong K K(\mathbb{C}, A)$ to $\left[\left(\tau \otimes \mathrm{id}_{n}\right)(p),\left(\gamma \otimes \mathrm{id}_{n}\right)(p)\right] \in K K(\mathbb{C}, B)$. More generally, if $D$ is a unital $\mathrm{C}^{*}$-algebra, then $\alpha_{D}=\left(\tau \otimes \mathrm{id}_{D}, \gamma \otimes \mathrm{id}_{D}\right)$ induces a map $\alpha_{*}: K_{0}(A \otimes D) \rightarrow K_{0}(B \otimes D)$. In particular $\alpha$ induces a map on $\underline{\mathbf{K}}(-)$.

Theorem 5.4. Let $A$ be a unital, separable, $C^{*}$-algebra and let $B$ be a unital $C^{*}$-algebra. Let $\alpha \in K K_{\mathrm{nuc}}(A, B)$. Assume that there exists a quasidiagonal strictly nuclear unital nuclearly absorbing representation $\gamma: A \rightarrow M(\mathscr{K}(H) \otimes B)$, and let $\gamma_{n}: A \rightarrow \mathbf{M}_{r_{n}}(B)$ be a quasidiagonalization of $\gamma$ by $\left(e_{n}\right)$ as in Definition 2.5.

For any $\underline{\mathbf{K}}$-triple $(\mathscr{P}, \mathscr{F}, \delta)$ there exist $N$ and a completely positive contraction

$$
\sigma: A \rightarrow \mathbf{M}_{2 r_{N}}(B)
$$

such that $\sigma$ and $\gamma_{N}$ are both nuclear, $\delta$-multiplicative on $\mathscr{F}$, and satisfy

$$
\sigma_{\#}(p)-\left(\gamma_{N}\right)_{\#}(p)=\alpha_{*}[p]
$$

for all $p \in \mathscr{P}$. We may arrange that $\sigma(1)$ and $\gamma_{N}(1)$ are both projections. 
Proof. By Lemma 3.1 we may represent $\alpha$ by the Cuntz pair $(\rho, \widehat{\theta})$ consisting of strictly nuclear representations. Recall that this entails that

$$
\rho(a)-\widehat{\theta}(a) \in \mathscr{K}(H) \otimes B
$$

for all $a \in A$.

Since $\widehat{\gamma}$ and $\widehat{\theta}$ are both nuclearly absorbing, we have $\widehat{\theta} \sim \widehat{\gamma}$ in the sense of Definition 2.1. Thus we get a sequence $u_{i} \in \mathscr{U}(M(\mathscr{K}(H) \otimes B))$ with

$$
\widehat{\gamma}(a)-u_{i} \widehat{\theta}(a) u_{i}^{*} \in \mathscr{K}(H) \otimes B, \quad\left\|\widehat{\gamma}(a)-u_{i} \widehat{\theta}(a) u_{i}^{*}\right\| \rightarrow 0 .
$$

Note that for each $i,\left(u_{i} \rho u_{i}^{*}, u_{i} \widehat{\theta} u_{i}^{*}\right)$ is a Cuntz pair representing $\alpha$. Then $X_{i}=\left(u_{i} \rho u_{i}^{*}, \widehat{\gamma}\right)$ is also a strictly nuclear Cuntz pair, and $\left[X_{i}\right]_{*}: \underline{\mathbf{K}}(A) \rightarrow \underline{\mathbf{K}}(B)$ converges pointwise to the group homomorphism induced by $\alpha$ as a consequence of Lemma 5.2. Fix $i$ large enough that $\left[X_{i}\right]_{*}[p]=\alpha_{*}[p]$ for each $p \in \mathscr{P}$.

Let $\tau=u_{i} \rho u_{i}^{*}$ and let $e_{n} \in \mathbf{M}_{r_{n}}(B)$ be as in the statement. Then $f_{n}=e_{n} \oplus e_{n} \in \mathbf{M}_{2 r_{n}}(B)$ is an approximate unit of projections which in the obvious sense quasidiagonalizes $\widehat{\gamma}$ into $\gamma_{n}: A \rightarrow \mathbf{M}_{2 r_{n}}(B)$. We set $\sigma_{n}=f_{n} \tau f_{n}$; the maps thus achieved are nuclear maps by Remark 2.6(ii).

Note now that

$$
\left[f_{n}, \tau(a)\right] \rightarrow 0, \quad f_{n}^{\perp}(\tau(a)-\widehat{\gamma}(a)) f_{n}^{\perp} \rightarrow 0
$$

for $a \in A$, since $\tau(a)-\widehat{\gamma}(a) \in \mathscr{K}(H) \otimes B$. We have $\mathscr{P} \subseteq C$ where

$$
C=\bigoplus_{m \leqslant M} A \otimes C(\mathbb{T}) \otimes C\left(W_{m}\right) \otimes \mathbf{M}_{K} .
$$

Therefore for $N$ large enough, $\sigma_{N}$ is $\delta$-multiplicative on $\mathscr{F}$ and

$$
\begin{gathered}
\left\|\left[f_{N} \otimes 1_{C},\left(\tau \otimes \operatorname{id}_{C}\right)(p)\right]\right\|<\frac{1}{9}, \quad\left\|\left[f_{N} \otimes 1_{C},\left(\widehat{\gamma} \otimes \operatorname{id}_{C}\right)(p)\right]\right\|<\frac{1}{9}, \\
\left\|f_{N}^{\perp} \otimes 1_{C}\left(\left(\tau \otimes \operatorname{id}_{C}\right)(p)-\left(\widehat{\gamma} \otimes \operatorname{id}_{C}\right)(p)\right) f_{N}^{\perp} \otimes 1_{C}\right\|<\frac{1}{9},
\end{gathered}
$$

for all $p \in \mathscr{P}$. By $\S 3.3$ and Lemma 5.3 we have

$$
\begin{aligned}
\left(\sigma_{N}\right)_{\#} & (p)-\left(\gamma_{N}\right)_{\#}(p) \\
& =\left[\chi_{0}\left(f_{N} \otimes 1_{C}\left(\tau \otimes \operatorname{id}_{C}\right)(p) f_{N} \otimes 1_{C}\right)\right]-\left[\chi_{0}\left(f_{N} \otimes 1_{C}\left(\widehat{\gamma} \otimes \operatorname{id}_{C}\right)(p) f_{N} \otimes 1_{C}\right)\right] \\
& =\left[\left(\tau \otimes \operatorname{id}_{C}\right)(p),\left(\widehat{\gamma} \otimes \operatorname{id}_{C}\right)(p)\right]=\left[X_{i}\right]_{*}[p]=\alpha_{*}[p] .
\end{aligned}
$$

By Lemma 5.1(iii) there is a sequence of unitaries $\left(v_{n}\right)$ in $M(\mathscr{K}(H) \otimes B)$ such that $\left[v_{n} \tau(1) v_{n}^{*}, f_{n}\right]=0$ and $\left\|v_{n}-1\right\| \rightarrow 0$ as $n \rightarrow \infty$. By replacing $\sigma_{n}=f_{n} \tau f_{n}$ by $\sigma_{n}=f_{n} v_{n} \tau v_{n}^{*} f_{n}$, we find that $\sigma_{n}(1)$ is a projection. Note that if $N$ is large enough, $\left(f_{N} \tau f_{N}\right)_{\#}(p)=\left(f_{N} v_{N} \tau v_{N}^{*} f_{N}\right)_{\#}(p)$, for $p \in \mathscr{P}$, by Lemma 3.13.

Next we specialize the result to the case of a quasidiagonal source $A$. An application of the general existence result for purely infinite $\mathrm{C}^{*}$-algebras can be found in Theorem 6.17.

We say, cf. Definition 2.10 , that $A$ is locally $R F D$ if for any finite set $\mathscr{F}$ and any $\varepsilon>0$, there exists an RFD subalgebra $A^{\prime}$ of $A$, containing all elements of $\mathscr{F}$ up to $\varepsilon$.

Theorem 5.5. Let $A$ and $B$ be unital $C^{*}$-algebras with $A$ quasidiagonal, and let $\alpha \in K K_{\mathrm{nuc}}(A, B)$. For any $\mathbf{K}$-triple $(\mathscr{P}, \mathscr{F}, \delta)$ there exist $N$ and completely 
positive nuclear contractions

$$
\sigma: A \rightarrow \mathbf{M}_{N}(B) \text { and } \mu: A \rightarrow \mathbf{M}_{N}\left(\mathbb{C} 1_{B}\right)
$$

which are $\delta$-multiplicative on $\mathscr{F}$ and satisfy

$$
\sigma_{\#}(p)-\mu_{\#}(p)=\alpha_{*}[p]
$$

for all $p \in \mathscr{P}$. We may arrange that $\sigma(1)$ and $\mu(1)$ are both projections. Moreover if $A$ is locally RFD and if $\varepsilon>0$ is given, we can arrange that there is a unital RFD subalgebra $D$ of $A$ such that $\mathscr{F} \subseteq_{\varepsilon} D$ and the restriction of $\mu$ to $D$ is a $*$-homomorphism.

Proof. Since $A$ is quasidiagonal, it has a quasidiagonal admissible scalar representation $\theta$. Note that, using projections $e_{n} \in \mathscr{K}(H) \otimes 1_{B}$ we obtain a quasidiagonalization consisting of maps $\theta_{n}: A \rightarrow \mathbf{M}_{r_{n}}\left(\mathbb{C} 1_{B}\right)$. The first part follows from Theorem 5.4, by taking $\mu=\theta_{n}$ for some large $n$.

In case $A$ is locally RFD, we find first a unital RFD subalgebra $D$ of $A$ such that $\mathscr{F} \subseteq_{\varepsilon_{1}} D$. Then we work with an admissible representation $\theta$ whose restriction to $D$ is a direct sum of finite-dimensional representations. It is then clear that one can choose the quasidiagonalization such that the restriction of $\theta_{n}$ to $D$ is a $*$-homomorphism for all $n$.

\section{Classification}

In this section, we present applications of the uniqueness and existence results to classification problems. The first part is devoted to a class of finite $\mathrm{C}^{*}$-algebras which allow a further refinement of the uniqueness result, leading to a complete classification of $\mathrm{C}^{*}$-algebras in this class having $K_{0}$-group $\mathbb{Q}$. In the last part, we show how the results also apply to reprove the classification theorem for purely infinite $\mathrm{C}^{*}$-algebras of Kirchberg and Phillips.

\subsection{Approximate unitary equivalence}

In a class of $\mathrm{C}^{*}$-algebras studied by $\mathrm{H}$. Lin it is possible to absorb the stabilization required in Theorem 4.15, leading to further improved uniqueness results. Furthermore, this class is contained in our class of admissible targets. In this section, we develop these points.

Definition 6.1 [35]. A simple unital $\mathrm{C}^{*}$-algebra $A$ is called tracially $A F$ (tracially approximately finite dimensional) if for any finite subset $\mathscr{F} \subseteq A$, any $\varepsilon>0$, and any non-zero projection $q \in A$, there is a projection $p \in A$, with $p \neq 1_{A}$, and there is a finite-dimensional $\mathrm{C}^{*}$-algebra $C \subseteq p^{\perp} A p^{\perp}$ with $p^{\perp} \in C$ such that

(i) $\|[p, a]\|<\varepsilon$ for all $a \in \mathscr{F}$,

(ii) $\operatorname{dist}\left(p^{\perp} a p^{\perp}, C\right)<\varepsilon$ for all $a \in \mathscr{F}$,

(iii) $u p u^{*} \leqslant q$ for some unitary $u \in A$.

EXAMPLE 6.2. The approximately (sub)homogeneous $\mathrm{C}^{*}$-algebras with real rank 0 classified in $[\mathbf{2 3}, 4.6]$ and $[\mathbf{1 5}, 9.1]$ are all tracially $\mathrm{AF}$ algebras. Also the class of examples of non-nuclear subalgebras of AF algebras constructed in [13] consists entirely of tracially AF algebras. 
The conditions (i)-(ii) from [42] imply quasidiagonality of $A$. Let us summarize a few other structural results on tracially $\mathrm{AF} \mathrm{C}^{*}$-algebras that we shall need. Lemmas 6.3 and 6.5 are from [35].

Lemma $6.3[35,3]$. Let $A$ be a simple unital tracially $A F C^{*}$-algebra. Then $A$ has real rank 0 and stable rank 1 , and $K_{0}(A)$ is weakly unperforated in the sense of [3]. When $p \in A$ is a projection and $n \in \mathbb{N}$, then both $p A p$ and $\mathbf{M}_{n}(A)$ are simple unital tracially $A F C^{*}$-algebras.

The following fact can be deduced from [53, 1.1(i)]. We give a direct argument for this more specialized claim.

LEMMA 6.4. Let $B$ be an infinite-dimensional unital separable simple $C^{*}$-algebra of real rank 0 and stable rank 1 , with $K_{0}(B)$ weakly unperforated. Then for any $n \geqslant 1$ and any non-zero projection $f \in B$ there are mutually orthogonal projections $e^{1}, \ldots, e^{n}$ and $r$ in $B$ such that $e^{1}+\ldots+e^{n}+r=1_{B}$ with $\left[e^{1}\right]=\ldots=\left[e^{n}\right]$ and $[r]<[f]$.

Proof. Let QT $(B)$ denote the normalized quasitraces on $B$. The image of the natural map $\rho_{0}: K_{0}(B) \rightarrow \operatorname{Aff}(\mathrm{QT}(B))$ is uniformly dense by [3, 6.9.3] and $K_{0}(B)$ has the strict ordering induced from $\rho_{0}$ by $[3,6.9 .2]$. If $e$ is a projection, we write $\widehat{e}=\rho_{0}(e)$. By simplicity, we find $N$ big enough such that $N[f]>[1]$. If $\varepsilon=1 / n N$, then $1 / n-\varepsilon>0$ and $n \varepsilon \widehat{1}<\widehat{f}$. Since the image of $\rho_{0}$ is uniformly dense, there is a projection $e \in B$ such that $(1 / n-\varepsilon) \hat{1}<\widehat{e}<1 / n \hat{1}$. Therefore $0<1-n \widehat{e}<n \varepsilon \widehat{1}<\widehat{f}$; hence

$$
0<[1]-n[e]<[f] .
$$

This is readily seen to imply the statement. Indeed if $d^{i} \in B$ are projections equivalent to $e$ then $d^{1} \oplus \ldots \oplus d^{n}$ is equivalent to a subprojection $d$ of $1_{B}$. If $r=1-d$, then $[r]=[1]-n[e]<[f]$.

The tracially $\mathrm{AF} \mathrm{C}^{*}$-algebras are suitable for classification because of the following factorization property.

Lemma 6.5 [35]. Let $A$ be a simple unital infinite-dimensional tracially $A F$ $C^{*}$-algebra. For any $n \geqslant 1$, any finite subset $\mathscr{F} \subseteq A$ and any $\varepsilon>0$, there are projections $p, q \in A$ with $p^{\perp} A p^{\perp} \cong \mathbf{M}_{n}(q A q)$ and $[p] \leqslant[q]$ and such that there exists an approximate factorization of $\mathrm{id}_{A}$,

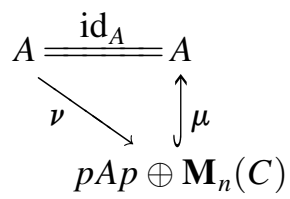

with $\|\mu \nu(a)-a\|<\varepsilon$ for $a \in \mathscr{F}$, where $C$ is a unital finite-dimensional $C^{*}$-subalgebra of qAq, $\nu(a)=$ pap $\oplus\left(\eta(a) \otimes 1_{n}\right)$ is $\varepsilon$-multiplicative on $\mathscr{F}$ with $\eta: A \rightarrow C$ a unital completely positive contractive map, and $\mu$ is a unital *-monomorphism whose restriction to $p A p$ is the natural inclusion. 
Proof. We include a proof of this result which is somewhat different from the original proof of Lin. We do not require $A$ to be nuclear. It also suffices to prove the statement with $8 \varepsilon$ instead of $\varepsilon$. Since $A$ is tracially AF, we find a projection $P \in A$ and a finite-dimensional $\mathrm{C}^{*}$-algebra $C$ with $P^{\perp} \in C \subset P^{\perp} A P^{\perp}$ such that for all $a \in \mathscr{F}$ we have

(i) $\|[P, a]\|<\varepsilon$,

(ii) $P^{\perp} a P^{\perp} \in \in_{\varepsilon} C$,

(iii) $(n+2)[P] \leqslant[1]$.

The idea of the proof is to find a unital embedding of $\mathbf{M}_{n}(\mathbb{C}) \oplus \mathbb{C}$ into the relative commutant of $C$ in $P^{\perp} A P^{\perp}$ such that the image of $\mathbb{C}$ is supported by a very small projection. Write $C \cong \mathbf{M}_{m(1)} \oplus \ldots \oplus \mathbf{M}_{m(k)}$ and let $e_{1}, \ldots, e_{k}$ be the minimal central projections of $C$. Let $B_{i}$ be the relative commutant of $e_{i} C e_{i} \cong \mathbf{M}_{m(i)}$ in $e_{i} A e_{i}$. Then $e_{i} A e_{i} \cong \mathbf{M}_{m(i)}\left(B_{i}\right)$ and hence $B_{i}$ is tracially $\mathrm{AF}$ as it is isomorphic to a corner of $A$. Let $f$ be a non-zero projection in $A$ with $(n+1) k[f]<[P]$. For each $1 \leqslant i \leqslant k$ we apply Lemma 6.4 for $B_{i}$. We obtain

$$
e_{i}=e_{i}^{1}+\ldots+e_{i}^{n}+r_{i}
$$

where $e_{i}^{j}$, for $1 \leqslant j \leqslant n$, are mutually equivalent projections in $B_{i}$ and $\left[r_{i}\right]<[f]$ in $K_{0}(A)$. Set $e^{j}=e_{1}^{j}+\ldots+e_{k}^{j}$ for $1 \leqslant j \leqslant n, r=r_{1}+\ldots+r_{k}$ and $e=e^{1}+\ldots+e^{n}$. Note that $P^{\perp}=e^{1}+\ldots+e^{n}+r$ with $e^{j}$ mutually equivalent in the relative commutant of $C$ in $P^{\perp} A P^{\perp}$. We have

$$
(n+1)[r]=(n+1)\left(\left[r_{1}\right]+\ldots+\left[r_{k}\right]\right)<(n+1) k[f]<[P] .
$$

Therefore

$$
(n+1)([P]+[r])<(n+2)[P] \leqslant[1]=[P]+n\left[e^{1}\right]+[r] ;
$$

hence $n([P]+[r])<n\left[e^{1}\right]$. By weak unperforation we get $[P+r] \leqslant\left[e^{1}\right]$. We are now ready to complete the proof. By Arveson's extension theorem [2], the identity map on $C$ extends to a completely positive contraction $E: A \rightarrow C$. We have $E(x)=x$ for $x \in C$. Hence $\|a-E(a)\| \leqslant 2 \operatorname{dist}(a, C)$ for $a \in A$. Using (i) and (ii) we have, for $a \in \mathscr{F}$,

$$
\begin{aligned}
a & \sim_{2 \varepsilon} P a P+P^{\perp} a P^{\perp} \sim_{2 \varepsilon} P a P+E\left(P^{\perp} a P^{\perp}\right) \\
& =P a P+E\left(P^{\perp} a P^{\perp}\right) r+E\left(P^{\perp} a P^{\perp}\right) e \\
& \sim_{4 \varepsilon}(P+r) a(P+r)+E\left(P^{\perp} a P^{\perp}\right) e .
\end{aligned}
$$

The last estimate follows by compressing the estimate $a \sim_{4 \varepsilon} P a P+E\left(P^{\perp} a P^{\perp}\right)$ by $P+r$. It follows that $a \sim_{8 \varepsilon}(P+r) a(P+r)+E\left(P^{\perp} a P^{\perp}\right) e$. We finish the proof by setting $p=P+r$ and $q=e^{1}$ and noting that $E\left(P^{\perp} a P^{\perp}\right) e$ is of the form $\eta(a) \otimes 1_{n}$ since $e=e^{1}+\ldots+e^{n}$ with $e^{j}$ mutually equivalent in the relative commutant of $C$ in $P^{\perp} A P^{\perp}$.

The uniqueness Theorems 4.12 and 4.15 apply to tracially AF algebras because of the following proposition.

Proposition 6.6. A simple unital infinite-dimensional tracially $A F C^{*}$-algebra is an admissible target algebra (of finite type). 
Proof. Let $B$ be a simple unital tracially AF $\mathrm{C}^{*}$-algebra. We get (i) and (ii) of Definition 4.9 by two results of Rieffel $[\mathbf{4 3}, \mathbf{4 4}]$. For (iii.1), note that if $n x \geqslant 0$ then $n x+n\left[1_{B}\right]>0$ and $x+\left[1_{B}\right]>0$ by weak unperforation. Finally, to prove (iv.1), assume that $\operatorname{dim}(B)=\infty$ and let $x$ and $n$ be given. The image of the natural map $\rho_{0}: K_{0}(B) \rightarrow \operatorname{Aff}(\mathrm{QT}(B))$ is uniformly dense by $[3,6.9 .3]$, so we can find $z \in K_{0}(B)$ with $\rho_{0}(x)-1<\rho_{0}(n z)<\rho_{0}(x)+1$. By $[3,6.9 .2]$ we have $x-\left[1_{B}\right] \leqslant n z \leqslant x+\left[1_{B}\right]$, so $y=x-n z$ will work.

THEOREM 6.7. Let $A$ be a simple unital, exact, separable tracially $A F$ $C^{*}$-algebra satisfying the UCT. Then for any finite subset $\mathscr{F} \subseteq A$ and any $\varepsilon>0$, there exists a $\underline{\mathbf{K}}$-triple $(\mathscr{P}, \mathscr{G}, \delta)$ with the following property. For any unital simple infinite-dimensional tracially $A F C^{*}$-algebra $B$, and any two unital nuclear completely positive contractions $\varphi, \psi: A \rightarrow B$ which are $\delta$-multiplicative on $\mathscr{G}$, with $\varphi_{\sharp}(p)=\psi_{\sharp}(p)$ for all $p \in \mathscr{P}$, there exists a unitary $u \in \mathscr{U}(B)$ such that

for all $a \in \mathscr{F}$.

$$
\left\|u \varphi(a) u^{*}-\psi(a)\right\|<\varepsilon
$$

Proof. Let us begin by outlining the proof. We first construct such a unitary in the special case where $\varphi$ and $\psi$ are nuclear $*$-homomorphisms agreeing on all of $\underline{\mathbf{K}}(A)$. This involves invoking Lin's factorization result, Lemma 6.5, to pass to another pair of $*$-homomorphisms $\bar{\varphi}$ and $\bar{\psi}$ which are of a special form. Because Lin's result only gives an approximate factorization, even though we start out with *-homomorphisms, our proof will take us to a setting where our uniqueness theorem for completely positive contractions, Theorem 4.15, is needed. The general case will follow in the same way that Theorem 4.12 implies Theorem 4.15 , by letting $n=0$ and $T=0$ in the proof of Theorem 4.15. We include a sketch for the benefit of the suspicious reader.

Part 1. Given $\mathscr{F}$ and $\varepsilon$, we are going to prove that whenever

(i) $B=\prod B_{i} / \sum B_{i}$ with each $B_{i}$ a unital simple infinite-dimensional tracially AF $C^{*}$-algebra (or $B$ itself is a unital simple infinite-dimensional tracially $\mathrm{AF} \mathrm{C}^{*}$-algebra),

(ii) $\varphi, \psi: A \rightarrow B$ are unital nuclear $*$-homomorphisms,

(iii) $\varphi_{*}=\psi_{*}: \underline{\mathbf{K}}(A) \rightarrow \underline{\mathbf{K}}(B)$,

then there exists $u \in \mathscr{U}(B)$ with $\left\|u \varphi(a) u^{*}-\psi(a)\right\|<\varepsilon$ for all $a \in \mathscr{F}$.

Let us thus fix $n, \mathscr{P}, \mathscr{G}$ and $\delta$ by applying Theorem 4.15 to $\mathscr{F}$ and $\frac{1}{3} \varepsilon$. Furthermore, let $p, q, C, \nu$ and $\mu$ be given by Lemma 6.5 such that $\nu$ is $\delta$-multiplicative on $\mathscr{G}$ and $\|\mu \nu(a)-a\|<\frac{1}{3} \varepsilon$ for all $a \in \mathscr{F}$.

Step 1a. Since $B$ has stable rank 1 by Lemma 6.3, it has cancellation of projections, and because $\varphi_{*}=\psi_{*}$ we may assume, after conjugating $\psi$ by a unitary in $B$, that the restrictions of $\varphi$ and $\psi$ to the finite-dimensional algebra $\mu\left(\mathbb{C} p \oplus \mathbf{M}_{n}\left(\mathbb{C} 1_{C}\right)\right)$ are equal. Applying $\mu$ to the matrix units of $\mathbf{M}_{n}\left(\mathbb{C} 1_{C}\right)$, we can define matrix units $\left(q_{i j}\right)$ in $A$, where $q=q_{11}=1_{C}$. Let

$$
e=\varphi(p)=\psi(p) \text { and } f_{i j}=\varphi\left(q_{i j}\right)=\psi\left(q_{i j}\right),
$$

abbreviating $f=f_{11}$. Invoking cancellation again, since $[p] \leqslant[q]$, we can find a projection $e_{0}$ and a unitary $v$ in $B$ with $v\left(e+e_{0}\right) v^{*}=f$. Let $g=e_{0} \oplus 1_{B} \in \mathbf{M}_{2}(B)$ 
and note that $[g]=(n+1) \cdot[f]$. Hence an isomorphism $\gamma: g \mathbf{M}_{2}(B) g \rightarrow \mathbf{M}_{n+1}(f B f)$ can be found. Denoting the matrix units of $\mathbf{M}_{n+1}(\mathbb{C} f)$ by $\widetilde{f}_{i j}$ with $0 \leqslant i, j \leqslant n$ we may choose $\gamma$ such that

$$
\gamma\left(\left[\begin{array}{cc}
e_{0} b_{0} e_{0} & \\
& e b e
\end{array}\right]\right)=\widetilde{f}_{00} v\left(e b e+e_{0} b_{0} e_{0}\right) v^{*} \widetilde{f}_{00}, \quad \gamma\left(\left[\begin{array}{ll}
0 & \\
& f_{i j}
\end{array}\right]\right)=\widetilde{f}_{i j} .
$$

Combining all of this, we get a $*$-homomorphism $\bar{\varphi}$ fitting in a diagram

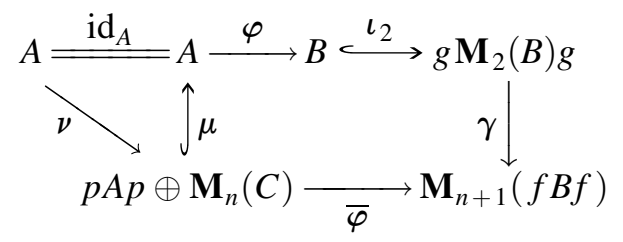

where $\iota_{2}$ sends $B$ into the $(2,2)$ corner of $g \mathbf{M}_{2}(B) g$. Identifying $\bar{\varphi}$ using (6.1) and (6.2) we see that for all $d \in p A p$ and $x \in \mathbf{M}_{n}(C)$,

$$
\bar{\varphi}(d \oplus x)=\varphi^{\prime \prime}(d) \oplus\left(\varphi^{\prime} \otimes \mathrm{id}_{n}\right)(x),
$$

where

$$
\varphi^{\prime}: C \rightarrow f B f \quad \text { and } \quad \varphi^{\prime \prime}: p A p \rightarrow f B f
$$

are defined as corestrictions of $\varphi$ and $\operatorname{Ad}(v) \varphi$, respectively. Furthermore, by symmetry of (6.1), the same procedure shows that $\bar{\psi}=\gamma \iota_{2} \psi \mu: p A p \oplus \mathbf{M}_{n}(C) \rightarrow$ $\mathbf{M}_{n+1}(f B f)$ has the form

$$
\bar{\psi}(d \oplus x)=\psi^{\prime \prime}(d) \oplus\left(\psi^{\prime} \otimes \mathrm{id}_{n}\right)(x)
$$

for all $d \in p A p$ and $x \in \mathbf{M}_{n}(C)$.

Step 1b. With $\iota_{f}: f B f \rightarrow B$ we clearly have $\left(\iota_{f} \varphi^{\prime}\right)_{*}=\left(\iota_{f} \psi^{\prime}\right)_{*}$ and $\left(\iota_{f} \varphi^{\prime \prime}\right)_{*}=$ $\left(\iota_{f} \psi^{\prime \prime}\right)_{*}$. But since $f$ is full in $B$ as the image of a full projection under a unital map, we find by [5] that $\iota_{f}$ induces an isomorphism from $\underline{\mathbf{K}}(f B f)$ to $\underline{\mathbf{K}}(B)$, so that $\left(\varphi^{\prime}\right)_{*}=\left(\psi^{\prime}\right)_{*}$ and $\left(\varphi^{\prime \prime}\right)_{*}=\left(\psi^{\prime \prime}\right)_{*}$. From this we may assume, after conjugating $\psi$ by a unitary of $f B f$, that $\varphi^{\prime}$ and $\psi^{\prime}$ agree on $C$. Thus the maps $\bar{\varphi} \nu$ and $\bar{\psi} \nu$ are of the form

$$
\begin{aligned}
& \bar{\varphi} \nu(a)=\left[\begin{array}{llll}
\varphi^{\prime \prime} \omega(a) & & & \\
& \varphi^{\prime} \eta(a) & & \\
& & \ddots & \\
& & & \varphi^{\prime} \eta(a)
\end{array}\right], \\
& \bar{\psi} \nu(a)=\left[\begin{array}{llll}
\psi^{\prime \prime} \omega(a) & & & \\
& \varphi^{\prime} \eta(a) & & \\
& & \ddots & \\
& & & \varphi^{\prime} \eta(a)
\end{array}\right],
\end{aligned}
$$

where we define $\omega: A \rightarrow p A p$ by $\omega(a)=$ pap. Note that $\omega$ is $\delta$-multiplicative on $\mathscr{G}$. Now $\varphi^{\prime \prime} \omega, \psi^{\prime \prime} \omega$ and $\varphi^{\prime} \eta$ are $\delta$-multiplicative on $\mathscr{G}$ and by Lemma 3.10 we have $\left(\varphi^{\prime \prime} \omega\right)_{\#}(p)=\left(\psi^{\prime \prime} \omega\right)_{\#}(p)$ for all $p \in \mathscr{P}$. Therefore, Theorem 4.15 applies to 
the triple of maps $\left(\varphi^{\prime \prime} \omega, \psi^{\prime \prime} \omega, \varphi^{\prime} \eta\right)$ if we can prove that $f B f$ is an admissible target. When $B$ itself is a tracially $\mathrm{AF} \mathrm{C}^{*}$-algebra, so is $f B f$ by Lemma 6.3 , and Proposition 6.6 applies. When $B=\prod B_{i} / \sum B_{i}$ we note that there are projections $f_{i} \in B_{i}$ such that $f B f$ is isomorphic to

$$
\prod f_{i} B_{i} f_{i} / \sum f_{i} B_{i} f_{i}
$$

and hence it is admissible of finite type by Lemma 3.4 since all the $f_{i} B_{i} f_{i}$ are. Thus, by Theorem 4.15 , there is a partial isometry $v \in \mathbf{M}_{n+1}(f B f)$ such that $v^{*} v=\bar{\varphi} \nu(1), v v^{*}=\bar{\psi} \nu(1)$ and $\left\|v \bar{\varphi} \nu(a) v^{*}-\bar{\psi} \nu(a)\right\|<\frac{1}{3} \varepsilon$ for all $a \in \mathscr{F}$. Since $\bar{\varphi} \nu(1)=\bar{\psi} \nu(1)=\gamma \iota_{2}(1)$ and $\gamma \iota_{2}(B)=\gamma \iota_{2}(1) \mathbf{M}_{n+1}(f B f) \gamma \iota_{2}(1)$, we have $v=\gamma \iota_{2}(u)$ for some unitary $u \in B$. Since $\gamma \iota_{2}$ is isometric, we obtain $\left\|u \varphi \mu \nu(a) u^{*}-\psi \mu \nu(a)\right\|<\frac{1}{3} \varepsilon$; hence $\left\|u \varphi(a) u^{*}-\psi(a)\right\|<\varepsilon$ for all $a \in \mathscr{F}$, since $\|\mu \nu(a)-a\|<\frac{1}{3} \varepsilon$ for all $a \in \mathscr{F}$.

Part 2. As the argument reducing to the case covered in Part 1 closely parallels that in the proof of Theorem 4.15, we only sketch it here. If the theorem is false, we can choose sequences $\mathscr{P}_{i}, \delta_{i}$ and $\mathscr{G}_{i}$ with the properties (i)-(iii) of that proof, and corresponding simple unital tracially $\mathrm{AF} \mathrm{C}^{*}$-algebras $B_{i}$ as well as unital completely positive contractions $\varphi_{i}, \psi_{i}: A \rightarrow B_{i}$ being $\delta_{i}$-multiplicative on $\mathscr{G}_{i}$, satisfying $\left(\varphi_{i}\right)_{\#}(p)=\left(\psi_{i}\right)_{\#}(p)$ for all $p \in \mathscr{P}_{i}$; yet

$$
\inf _{u \in \mathscr{U}(B)} \max _{a \in \mathscr{F}}\left\|u \varphi_{i}(a) u^{*}-\psi_{i}(a)\right\| \geqslant \varepsilon .
$$

Define $\dot{\Phi}, \dot{\Psi}: A \rightarrow \prod B_{i} / \sum B_{i}$ and check as in the proof of Theorem 4.15 that $\dot{\Phi}$ and $\dot{\Psi}$ are $*$-homomorphisms inducing the same map on $\underline{\mathbf{K}}(A)$. Since (i)-(iii) of Part 1 are met, we conclude from the first part of the proof that there is a unitary $U \in \mathscr{U}\left(\prod B_{i} / \sum B_{i}\right)$ such that $\left\|U \dot{\Phi}(a) U^{*}-\dot{\Psi}(a)\right\|<\varepsilon$ for all $a \in \mathscr{F}$. Lifting $U$ to a unitary $\left(u_{i}\right) \in \prod B_{i}$ and projecting, we get the desired contradiction.

Notes 6.8. As noted above, Definition 6.1 is due to H. Lin. It was motivated by a result of Popa [42] and the classification theory of AH algebras [23, 4.6; 15, 9.1]. Definition 6.1 is in fact a version of the original definition, slightly simplified for the class of simple algebras. To correlate this with [35] one compares to $[35,3.7]$ and notes that since Popa's conditions imply the (SP) property (all hereditary subalgebras have a non-zero projection), it suffices to find a $p$ inside a generic corner rather than inside a generic hereditary subalgebra.

\subsection{Classification results}

We begin this section by presenting a shape-type isomorphism result for simple tracially $\mathrm{AF} \mathrm{C}^{*}$-algebras. Note that in this setting, there is no need to appeal to our existence results, as the $K K$-classes are represented by completely positive contractions from the outset. We then prove, this time using both existence and

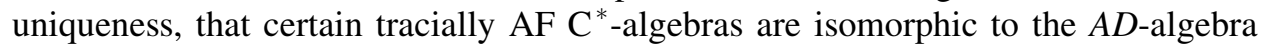
with the same $K$-theory. This leads to our main classification result, Theorem 6.13. We refer the reader to [8] for the basics of asymptotic morphisms.

Theorem 6.9. Let $A$ and $B$ be two unital, separable, nuclear, simple tracially $A F C^{*}$-algebras satisfying the UCT. Suppose that there are unital asymptotic 
morphisms $\varphi=\left(\varphi_{t}\right): A \rightarrow B$ and $\psi=\left(\psi_{t}\right): B \rightarrow A$ such that $\varphi_{*}: \underline{\mathbf{K}}(A) \rightarrow \underline{\mathbf{K}}(B)$ is bijective and $\varphi_{*}^{-1}=\psi_{*}$. For instance, this condition is satisfied if $A$ is homotopy equivalent, or just shape equivalent, to $B$. Then $A$ is isomorphic to $B$.

Proof. We may assume that both $A$ and $B$ are infinite dimensional. We use Theorem 6.7 repeatedly to find an increasing sequence of positive numbers $t_{n} \in(0,1)$ and sequences of unitaries $u_{2 n-1} \in A$ and $u_{2 n} \in B$, with $n \geqslant 1$ such that the following diagram is a two-sided approximate intertwining in the sense of Elliott [22]:

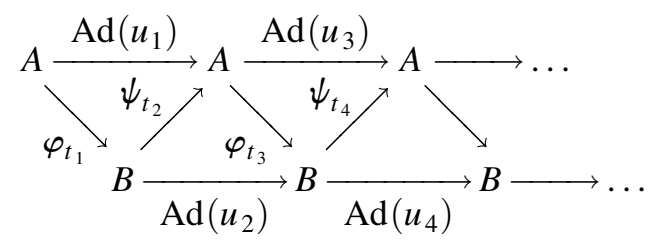

If $r$ is a positive integer, we denote by $\square_{r}^{\sim}$ the $\mathrm{C}^{*}$-algebra of continuous functions $f:[0,1] \rightarrow \mathbf{M}_{r}(\mathbb{C})$ such that $f(0), f(1) \in \mathbb{C} 1_{r}$. We denote by $\mathbb{q}_{r}$ the subalgebra of functions vanishing at 0 . Let $\mathscr{D}$ be a class of algebras $B$ of the form $B=B_{1} \oplus \ldots \oplus B_{n}$ where each $B_{i}$ is either a circle algebra $\mathbf{M}_{k}\left(C\left(S^{1}\right)\right)$ or a dimension-drop algebra $\mathbf{M}_{k}\left(\nabla_{r}^{\sim}\right)$. An $A D$-algebra is a $\mathrm{C}^{*}$-algebra which is isomorphic to an inductive limit of a sequence of $\mathrm{C}^{*}$-algebras in $\mathscr{D}$.

Lemma 6.10. Let $D=\mathbb{1}_{n}$ or $D=C_{0}(\mathbb{R})$. Let $E$ be a simple unital tracially $A F$ algebra and let $f$ be a non-zero projection in $E$. Then the map $[D, f E f] \rightarrow K K(D, E)$ is surjective.

Proof. Let $n=1$ if $D=C_{0}(\mathbb{R})$. The proof uses the following series of facts.

(i) $[19] \stackrel{\lim }{\longrightarrow}\left[D, \mathbf{M}_{k}(E)\right]=K K(D, E)$.

(ii) ([17] when $D=\mathbb{\square}_{n}$ ) If $C$ is a finite-dimensional $C^{*}$-algebra, and $\eta: D \rightarrow C$ is a $*$-homomorphism, then the map $d \mapsto \eta(d) \otimes 1_{n}$ from $D \rightarrow \mathbf{M}_{n}(C)$ is null homotopic.

(iii) [38] There is a finite subset $\mathscr{F} \subseteq D$ and there is $\varepsilon>0$ such that if $\alpha, \beta: D \rightarrow B$ are two $*$-homomorphisms satisfying $\|\alpha(d)-\beta(d)\|<\varepsilon$, then $\alpha$ is homotopic to $\beta$.

(iv) [38] For any finite subset $\mathscr{F} \subseteq D$ and $\varepsilon>0$ there is a finite subset $\mathscr{F}_{1} \subseteq D$ and there is $\varepsilon_{1}>0$ such that if $\alpha: D \rightarrow B$ is any completely positive contractive map which is $\varepsilon_{1}$-multiplicative on $\mathscr{F}_{1}$, then there exists a *-homomorphism $\beta: D \rightarrow B$ with $\|\alpha(d)-\beta(d)\|<\frac{1}{2} \varepsilon$ for all $d \in \mathscr{F}$.

Fix $\mathscr{F}$ and $\varepsilon$ as in (iii) and let $\mathscr{F}_{1}$ and $\varepsilon_{1}$ be given by (iv). We may assume that $\mathscr{F} \subseteq \mathscr{F}_{1}$ and $\varepsilon>\varepsilon_{1}$. Let $x \in K K(D, E)$. Then by (i), $x$ can be represented by some $*$-homomorphism $\gamma: D \rightarrow \mathbf{M}_{k}(E)$. With $A=\mathbf{M}_{k}(E), A$ is a simple unital tracially $\mathrm{AF} \mathrm{C}^{*}$-algebra by Lemma 6.3 . Since $E$ is simple, there is $m \geqslant 1$ such that $\left[1_{E}\right] \leqslant m[f]$. Consider an approximation of $\mathrm{id}_{A}$ provided by Lemma 6.5 applied for the set $\gamma\left(\mathscr{F}_{1}\right), \frac{1}{2} \varepsilon_{1}$, and the integer $m n k$ :

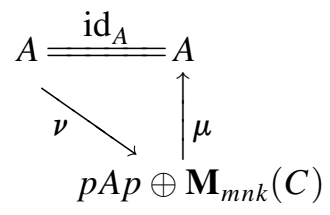


Therefore

$$
\|\mu \nu(a)-a\|<\frac{1}{2} \varepsilon_{1}
$$

for $a \in \gamma\left(\mathscr{F}_{1}\right)$, where $C$ is a unital finite-dimensional $\mathrm{C}^{*}$-subalgebra of $q A q$, $\nu(a)=\omega(a) \oplus\left(\eta(a) \otimes 1_{m n k}\right), \omega(a)=$ pap, $\nu$ is $\varepsilon_{1}$-multiplicative on $\gamma\left(\mathscr{F}_{1}\right)$ and $\eta: A \rightarrow C$ is a unital completely positive contractive map. We may arrange that $\mu(p) \leqslant f$. Indeed, from $[p] \leqslant[q]$ and $[p]+m n k[q]=1$ we see that $(m n k+1)[p] \leqslant[1]$. Since $\mu(1)=1_{A}$, we obtain

$$
(m n k+1)[\mu(p)] \leqslant\left[1_{A}\right]=n\left[1_{E}\right] \leqslant m n k[f] .
$$

Hence $[\mu(p)] \leqslant n[\mu(p)]<[f]$ since $K_{0}(E)$ is weakly unperforated in the sense of [3] by Lemma 6.3. After conjugating $\mu$ by a suitable unitary, we obtain $\mu(p) \leqslant f$.

Let us observe that $\omega \gamma$ and $\eta \gamma$ are $\varepsilon_{1}$-multiplicative on $\mathscr{F}_{1}$. By (iv) there are $*$-homomorphisms $\omega^{\prime}: D \rightarrow p A p$ and $\eta^{\prime}: D \rightarrow C$ such that if we set $\nu^{\prime}=\omega^{\prime} \oplus\left(\eta^{\prime} \otimes 1_{m n}\right)$, then

$$
\left\|\nu \gamma(d)-\nu^{\prime}(d)\right\|<\frac{1}{2} \varepsilon
$$

for all $d \in \mathscr{F}$. From (6.3) we have $\|\mu \nu \gamma(d)-\gamma(d)\|<\frac{1}{2} \varepsilon_{1}$ for $d \in \mathscr{F}_{1}$. Combining this with (6.4) we get

$$
\left\|\mu \nu^{\prime}(d)-\gamma(d)\right\|<\frac{1}{2}\left(\varepsilon_{1}+\varepsilon\right)<\varepsilon
$$

for all $d \in \mathscr{F}_{1} \cap \mathscr{F}=\mathscr{F}$. By (iii) this implies that $\gamma$ is homotopic to $\mu \nu^{\prime}$. By (ii), $\nu^{\prime}$ is homotopic to $\omega^{\prime}$ so that $\gamma$ is homotopic to $\mu \omega^{\prime}$. We conclude the proof by observing that the image of $\mu \omega^{\prime}$ is contained in $f E f$ since $\mu \omega^{\prime}(1)=\mu(p) \leqslant f$.

Lemma 6.11. Let $A$ be a unital $C^{*}$-algebra with $\left(K_{0}(A),\left[1_{A}\right]\right)=(\mathbb{Q}, 1)$. Any finite set of projections $\mathscr{P}_{0} \subseteq A \otimes \mathscr{K}$ can be augmented to a $K_{0}$-triple $\left(\mathscr{P}_{0}, \mathscr{G}, \delta\right)$ with the property that for any unital completely positive contraction $\varphi: A \rightarrow A$ which is $\delta$-multiplicative on $\mathscr{G}$, one has $\varphi_{\sharp}(p)=[p]$ for all $p \in \mathscr{P}_{0}$.

Proof. We may write $[p]=(r / s)\left[1_{A}\right]$, so $s \cdot p \oplus m \cdot 1_{A} \sim(r+m) \cdot 1_{A}$ for some $m \geqslant 0$. When $\varphi$ is sufficiently multiplicative, we have

$$
s \varphi_{\#}(p)+m\left[1_{A}\right]=\varphi_{\#}\left(s \cdot p \oplus m \cdot 1_{A}\right)=\varphi_{\#}\left((r+m) \cdot 1_{A}\right)=(r+m)\left[1_{A}\right] .
$$

The following theorem generalizes a result of Lin [35] by the fact that it allows non-zero (countable) $K_{1}$-groups.

THeORem 6.12. Let A be a unital, separable, nuclear and simple tracially AF $C^{*}$-algebra satisfying the UCT and suppose that $K_{0}(A) \cong \mathbb{Q}$ as ordered groups. Then $A$ is isomorphic to an AD-algebra.

Proof. Clearly $A$ is infinite dimensional, and we may assume that $\left(K_{0}(A),\left[1_{A}\right]\right) \cong(\mathbb{Q}, 1)$ as ordered pointed groups. For any finite subset $\mathscr{F} \subseteq A$ and any $\varepsilon>0$ we will find an algebra $B \in \mathscr{D}$, and a $*$-homomorphism $\beta: B \rightarrow A$ such that $\mathscr{F} \subseteq_{\varepsilon} \beta(B)$. This will prove the theorem as all elements of the class $\mathscr{D}$ (introduced before Lemma 6.10) are semiprojective [38]. As noted in Remark 4.16, applying Theorem 4.15 to fixed $\mathscr{F}$ and $\varepsilon$ associated to $C^{*}$-algebras $A$ and $B$ with torsion-free divisible $K_{0}$-groups results in a $K_{*}$-triple $(\mathscr{P}, \mathscr{G}, \delta)$ rather than a general $\underline{\mathbf{K}}$-triple. 
Let $\left(\mathscr{P}_{0}, \mathscr{G}_{0}, \delta_{0}\right)$ and $\left(\mathscr{V}, \mathscr{P}_{1}, \delta_{1}\right)$ be a $K_{0}$-triple and a $K_{1}$-triple, respectively, given by Lemma 3.11 for the input $K_{*}$-triple $(\mathscr{P}, \mathscr{G}, \delta)$. Let $\left(\mathscr{P}^{\prime}, \mathscr{G}^{\prime}, \delta^{\prime}\right)$ be a $K_{*}$-triple given by Lemma 3.12 for the input triples $\left(\mathscr{P}, \mathscr{G}_{0}, \delta_{0}\right)$ and $\left(\mathscr{V}, \mathscr{G}_{1}, \delta_{1}\right)$. We also may assume that $\mathscr{G}^{\prime}$ and $\delta^{\prime}$ satisfy the conclusion of Lemma 6.11 applied for the unital $\mathrm{C}^{*}$-algebra $A$ and the set of projections $\mathscr{P}_{0}$.

By [22] there are an $A D$-algebra $D$ and a group isomorphism $\kappa: K_{1}(A) \rightarrow K_{1}(D)$. By Theorem 5.5 there exist completely positive contractive maps $\sigma: A \rightarrow M_{N}(D)$ and $\mu: A \rightarrow M_{N}\left(\mathbb{C} 1_{D}\right)$ which are $\delta^{\prime}$-multiplicative on $\mathscr{G}^{\prime}$ and $\sigma_{\#}\left(p^{\prime}\right)-\mu_{\#}\left(p^{\prime}\right)=\kappa\left[p^{\prime}\right]$ for all $p^{\prime} \in \mathscr{P}^{\prime}$. Here $\kappa$ is regarded as an element of $\operatorname{Hom}\left(K_{*}(A), K_{*}(B)\right) \cong$ $\operatorname{Hom}_{\Lambda}(\underline{\mathbf{K}}(A), \underline{\mathbf{K}}(B))$. By the choice of the $K_{*}$-triple $\left(\mathscr{P}^{\prime}, \mathscr{G}^{\prime}, \delta^{\prime}\right)$ we have $\sigma_{\#}(u)-\mu_{\#}(u)=\kappa[u]$ for all unitaries $u \in \mathscr{V}$. Note that $\mu_{\#}(u)=0$ since $K_{1}(\mathbb{C})=0$, so that we have $\sigma_{\sharp}(u)=\kappa[u]$ for all $u \in \mathscr{V}$.

Recall that $\sigma\left(1_{A}\right)=q$ is a projection, so that if we set $B=q M_{N}(D) q$, then $\sigma: A \rightarrow B$ is a unital map. Write $B$ as the inductive limit of an increasing sequence of algebras $B_{k} \in \mathscr{D}$, and let $j_{k}: B_{k} \rightarrow B$ be the inclusion map. Using the ChoiEffros theorem as in Lemma 4.2 of [16], we find a sequence of completely positive contractive maps $\eta_{k}: B \rightarrow B_{k}$ such that $j_{k} \eta_{k}$ converges to $\mathrm{id}_{B}$ in the point-norm topology. Choose $k$ large enough so that

$$
\sigma_{\#}(u)=\left(j_{k} \eta_{k} \sigma\right)_{\sharp}(u)
$$

for all $u \in \mathscr{V}$.

Consider the group morphism $\kappa^{-1}\left(j_{k}\right)_{*}: K_{1}\left(B_{k}\right) \rightarrow K_{1}(A)$. There is a unital *-homomorphism $\gamma: B_{k} \rightarrow A$ such that $\gamma_{*}=\kappa^{-1}\left(j_{k}\right)_{*}$, obtained as follows. Write $B_{k}=\mathbf{M}_{\ell(1)}\left(B_{k_{1}}^{\prime}\right) \oplus \ldots \oplus \mathbf{M}_{\ell(r)}\left(B_{k_{r}}^{\prime}\right)$ where the $B_{k_{i}}^{\prime}$ are either $C\left(S^{1}\right)$ or $\widetilde{\mathbb{q}_{n(i)}}$. Let $q_{1}+\ldots+q_{r}=1_{A}$ be a partition of $1_{A}$ by non-zero projections. We have $K_{0}\left(q_{i} A q_{i}\right) \cong K_{0}(A) \cong \mathbb{Q}$ as ordered groups. Therefore we find mutually orthogonal projections $p_{i}$ such that $\ell(i)\left[p_{i}\right] \cong\left[q_{i}\right]$. Since $A$ has cancellation of projections, we find a unital inclusion

$$
\bigoplus_{i=1}^{r} M_{\ell(i)}\left(p_{i} A p_{i}\right) \subseteq A
$$

Using Lemma 6.10 we find unital $*$-homomorphisms $\gamma_{i}: B_{i} \rightarrow p_{i} A p_{i}$ such that $\gamma=\bigoplus_{i=1}^{r}\left(\gamma_{i} \otimes \mathrm{id}_{\ell(i)}\right)$ has the desired property.

Next we want to show that $\gamma \circ\left(\eta_{k} \sigma\right)$ gives an approximate factorization of $\operatorname{id}_{A}$ on $K_{*}(A)$. More precisely we want $\left(\gamma \eta_{k} \sigma\right)_{\sharp}(p)=[p]$ for all $p \in \mathscr{P}$. By virtue of our choice of the $K_{0}$ - and $K_{1}$-triples above, it suffices to show that $\left(\gamma \eta_{k} \sigma\right)_{\#}\left(p_{0}\right)=\left[p_{0}\right]$ for all $p_{0} \in \mathscr{P}_{0}$ and $\left(\gamma \eta_{k} \sigma\right)_{\#}(u)=[u]$ for all $u \in \mathscr{V}$. Using Lemma 3.10 twice, the definition of $\gamma$ and (6.5), we have

$$
\begin{aligned}
\left(\gamma \eta_{k} \sigma\right)_{\#}(u) & =\gamma_{*}\left(\eta_{k} \sigma\right)_{\#}(u)=\kappa^{-1}\left(j_{k}\right)_{*}\left(\eta_{k} \sigma\right)_{\#}(u) \\
& =\kappa^{-1}\left(j_{k} \eta_{k} \sigma\right)_{\#}(u)=\kappa^{-1} \sigma_{\#}(u)=[u]
\end{aligned}
$$

for all $u \in \mathscr{V}$. It remains to check that $\left(\gamma \eta_{k} \sigma\right)_{\#}\left(p_{0}\right)=\left[p_{0}\right]$ for all $p_{0} \in \mathscr{P}_{0}$, but this follows from Lemma 6.11 by our choice of the $\mathscr{G}^{\prime}$ and $\delta^{\prime}$.

Define $\alpha=\eta_{k} \sigma: A \rightarrow B_{k}$. We have seen that $(\gamma \alpha)_{\#}(p)=[p]$ for all $p \in \mathscr{P}$. Therefore by Theorem 4.15 there is a unitary $u \in A$ such that if $\beta=\operatorname{Ad}(u) \circ \gamma$, then $\|\beta \alpha(a)-a\|<\varepsilon$ for all $a \in \mathscr{F}$, and hence $\mathscr{F} \subseteq_{\varepsilon} \beta(B)$. 
THEOREM 6.13. Let $A$ and $B$ be unital, separable, nuclear and simple tracially $A F$ $C^{*}$-algebras satisfying the UCT. Suppose that $\left(K_{0}(A),\left[1_{A}\right]\right) \cong\left(K_{0}(B),\left[1_{B}\right]\right) \cong(\mathbb{Q}, 1)$ as ordered groups, and $K_{1}(A) \cong K_{1}(B)$. Then $A$ is isomorphic to $B$.

Proof. By Theorem 6.12 both $A$ and $B$ are isomorphic to simple $A D$-algebras of real rank 0 . These are classified by their $K$-theory data as proved by Elliott [22].

Notes 6.14. Lin proved Theorem 6.12 for $K_{1}(A)=0$ in [35]. He subsequently, independently from and at about the same time as the present work, generalized his result to allow general $K_{1}$-groups in [36]. This paper also attempts to give classification of $\mathrm{C}^{*}$-algebras with other $K_{0}$-groups.

\subsection{Purely infinite $C^{*}$-algebras}

The purpose of this section is to demonstrate how our methods can be applied to give the classification result of Kirchberg and Phillips starting from three basic, albeit deep, structural results about purely infinite $\mathrm{C}^{*}$-algebras. The methods used here are very similar to those used in the finite case, with Cuntz' algebra $\mathcal{O}_{2}$ playing the role of $\mathbf{M}_{n}(\mathbb{C})$. The exposition will emphasize this similarity. Our starting point is the following observation.

Proposition 6.15. A purely infinite simple unital $C^{*}$-algebra is an admissible target (of infinite type).

Proof. A purely infinite simple $\mathrm{C}^{*}$-algebra $A$ has real rank 0 as noted in [52], and as seen in [10], the canonical maps from $\operatorname{Proj}(A)$ and $\mathscr{U}(A)$ to $K_{0}(A)$ and $K_{1}(A)$ are surjective. Furthermore, it is also noted there that if $p, q \in A$ are non-zero projections, then

$$
[p]=[q] \Longrightarrow p \sim q,
$$

proving the remaining requirement.

To make it very clear exactly how much else we need to import from the theory of this class of $\mathrm{C}^{*}$-algebras we collect the required results below.

(I) $[31,2.8]$ Any exact, separable and unital $\mathrm{C}^{*}$-algebra embeds unitally into $\mathcal{O}_{2}$.

(II) $[45,3.6]$ Let $B$ be an admissible target algebra. If $\varphi, \psi: \mathcal{O}_{2} \rightarrow B$ is a pair of unital $*$-homomorphisms, then for any finite set $\mathscr{F} \subseteq \mathcal{O}_{2}$ and any $\varepsilon>0$ there exists $u \in \mathscr{U}(B)$ with $\left\|u \varphi(a) u^{*}-\psi(a)\right\|<\varepsilon$ for all $a \in \mathscr{F}$.

(III) (A variation of $[40,2.4]$ ) Let $A$ be a purely infinite simple nuclear separable unital $\mathrm{C}^{*}$-algebra. For any $n \geqslant 1$, any finite subset $\mathscr{F} \subseteq A$ and any $\varepsilon>0$, there are a projection $p \in A$ and a unital inclusion $\mathbf{M}_{n}\left(\mathcal{O}_{2}\right) \subset p^{\perp} A p^{\perp}$ such that there exists an approximate factorization of $\mathrm{id}_{A}$,

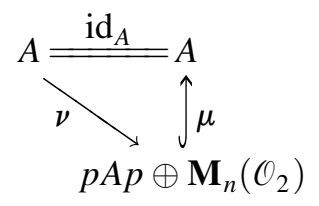


where $\|\mu \nu(a)-a\|<\varepsilon$ for $a \in \mathscr{F}, \nu(a)=\operatorname{pap} \oplus\left(\eta(a) \otimes 1_{n}\right)$ is $\varepsilon$-multiplicative on $\mathscr{F}$ with $\eta: A \rightarrow \mathrm{O}_{2}$ a unital completely positive contractive map, and $\mu$ is a unital $*$-monomorphism whose restriction to $p A p$ is the natural inclusion.

REMARK 6.16. We have rephrased (II) and (III) slightly to suit our needs. Rørdam requires that $B$ satisfies

$$
\mathscr{U}(B) / \mathscr{U}_{0}(B) \cong K_{1}(B)
$$

and has finite exponential length [39], but this follows from Definition 4.9 by [32]. Also, Phillips proves (III) only for $n=1$, but since it is easy to prove directly, by splitting the unit of $\mathcal{O}_{2}$ into matrix units, that $\mathbf{M}_{n}\left(\mathcal{O}_{2}\right) \cong \mathcal{O}_{2}$, we may replace $\mathcal{O}_{2}$ by $\mathbf{M}_{n}\left(\mathcal{O}_{2}\right)$ without loss of generality (and without using the theorem we are aiming for).

Theorem 6.17. Let $A$ and $B$ be unital $C^{*}$-algebras with $A$ exact and separable and $B$ containing a unital copy of $\mathcal{O}_{2}$, and let $\alpha \in K K_{\text {nuc }}(A, B)$. Then for any $\underline{\mathbf{K}}$-triple $(\mathscr{P}, \mathscr{F}, \delta)$ there exists a nuclear completely positive contraction $\sigma: A \rightarrow B$ which is $\delta$-multiplicative on $\mathscr{F}$ and satisfies $\sigma_{\sharp}(p)=\alpha_{*}[p]$ for all $p \in \mathscr{P}$. We may arrange that $\sigma(1)$ is a projection.

Proof. By (I), $A$ embeds unitally into $\mathcal{O}_{2}$, so with $\iota$ defined as the composite

$$
A \longleftrightarrow \mathrm{O}_{2} \longleftrightarrow B
$$

we obtain a unital full embedding. By Theorem 2.22, the representation $d_{\iota}$ is absorbing, and it is clearly also quasidiagonal as it commutes with the projections $e_{n}=n \cdot 1_{B}$. By Theorem 5.4, we find $N$ and $\sigma: A \rightarrow \mathbf{M}_{2 N}(B)$ such that

$$
(\sigma)_{\#}(p)-\left(\gamma_{N}\right)_{\#}(p)=\alpha_{*}[p]
$$

where $\gamma_{N}$ is a $*$-homomorphism of the form

$$
\gamma_{N}(a)=0 \oplus N \cdot \iota(a),
$$

so that $\left(\gamma_{N}\right)_{*}=0$ by the fact that $\iota$ factors through $\mathcal{O}_{2}$. Thus $(\sigma)_{\#}=\alpha_{*}$ on $\mathscr{P}$, and assuming, as we may, that $\sigma(1)$ is a projection $e$, we find that $e \in \mathbf{M}_{2 N+1}(B)$ is a subprojection of $(2 N) \cdot 1_{B}$ which is equivalent in $\mathbf{M}_{2 N+1}(B)$ to a subprojection of $1_{B}=1_{\mathcal{O}_{2}}$ via some unitary $u$. We may hence replace $\sigma$ by $u \sigma u^{*}: A \rightarrow B$ inducing the same partial map on $\mathscr{P}$.

REMARK 6.18. If $B$ is simple purely infinite and $\alpha_{*}[1]=[1]$, then after taking $1 \in \mathscr{P}$ we may arrange that $\sigma$ is unital. Indeed, since $\sigma(1)=e$ is a projection and $\sigma_{\sharp}[1]=\alpha_{*}[1]=[1]$, we have $[1-e]=0$, and hence $(1-e) B(1-e)$ contains a unital copy of $\mathcal{O}_{2}$. Let $J: A \rightarrow(1-e) B(1-e)$ be a unital embedding with image contained in this copy of $\mathcal{O}_{2}$. Replacing $\sigma$ by $\sigma \oplus J$ we see that $\sigma_{\#}=(\sigma \oplus J)_{\#}$ on $\mathscr{P}$ and $\sigma \oplus J$ is unital.

REMARK 6.19. Kirchberg proved a stronger form of the previous theorem where $\alpha$ is lifted to a $*$-monomorphism.

THEOREM 6.20. Let $A$ be a purely infinite simple separable unital nuclear $C^{*}$-algebra satisfying the UCT. Then for any finite subset $\mathscr{F} \subseteq A$ and any $\varepsilon>0$, 
there exists a $\underline{\mathbf{K}}$-triple $(\mathscr{P}, \mathscr{G}, \delta)$ with the following property. For any purely infinite simple unital $C^{*}$-algebra $B$, and any two unital nuclear completely positive contractions $\varphi, \psi: A \rightarrow B$ which are $\delta$-multiplicative on $\mathscr{G}$, with $\varphi_{\#}(p)=\psi_{\#}(p)$ for all $p \in \mathscr{P}$, there exists a unitary $u \in \mathscr{U}(B)$ such that $\left\|u \varphi(a) u^{*}-\psi(a)\right\|<\varepsilon$ for all $a \in \mathscr{F}$.

Proof. The proof follows closely that of Theorem 6.7, and we are only going to indicate the changes needed. Here Part 1 of the proof deals with a pair of *-homomorphisms into $B=\prod B_{i} / \sum B_{i}$ with each $B_{i}$ a purely infinite simple $\mathrm{C}^{*}$-algebra. Applying first Theorem 4.15 and then (III) we get $n, \mathscr{P}, \mathscr{G}, \delta, p, q, \nu$ and $\mu$ as in that proof. Then Step 1a applies verbatim as soon as one notes that because they are all non-zero, one has cancellation on all the projections in play by the result of Cuntz mentioned above. In Step $1 \mathrm{~b}$ one applies (II) to obtain a unitary of $f B f$ which conjugates $\psi^{\prime}$ to $\varphi^{\prime}$ to within $\frac{1}{3} \varepsilon$ on $\mathscr{F}$. This suffices to achieve the desired conclusion by the argument given in Theorem 4.15. Furthermore, to show that $f B f$ is admissible of infinite type, one uses the same argument to reduce to the case of showing that a corner of a purely infinite $\mathrm{C}^{*}$-algebra is also admissible of infinite type. This is clear since it is itself purely infinite. Finally, Part 2 of the proof carries through verbatim because of Proposition 6.15.

THeOREM $6.21[30,41]$. Let $A$ and $B$ be purely infinite simple separable unital nuclear $C^{*}$-algebras satisfying the UCT. Then any isomorphism $\kappa:\left(K_{*}(A),\left[1_{A}\right]\right) \rightarrow\left(K_{*}(B),\left[1_{B}\right]\right)$ is induced by a *-isomorphism.

Proof. We may assume that $A$ and $B$ are in the standard form, that is $1_{A}$ and $1_{B}$ both represent the zero class in their respective $K_{0}$-groups. It follows by [10] that $A$ and $B$ both contain unital copies of $\mathcal{O}_{2}$. We may thus apply the existence result Theorem 6.17 in conjunction with Remark 6.18 to get unital maps $\sigma_{i}: A \rightarrow B$ and $\tau_{i}: B \rightarrow A$ which are increasingly multiplicative on larger and larger sets, and induce $\kappa$ and $\kappa^{-1}$, respectively, on larger and larger subsets of $\underline{\mathbf{K}}(A)$ and $\underline{\mathbf{K}}(B)$. Arranging this appropriately, we may conclude by the uniqueness result Theorem 6.20 that unitaries $u_{i}$ and $v_{i}$ exist making

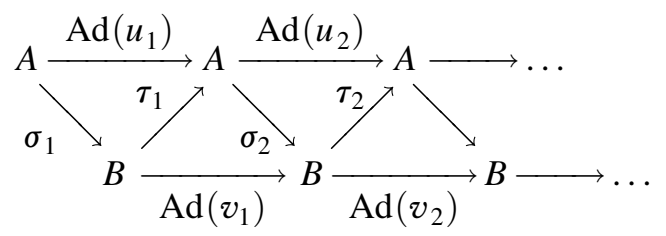

an approximate intertwining in the sense of Elliott [22].

\section{References}

1. C. A. Akemann, J. Anderson and G. K. Pedersen, 'Excising states in $\mathrm{C}^{*}$-algebras', Canad. J. Math. 38 (1986) 1239-1260.

2. W. Arveson, 'Notes on extensions of C*-algebras', Duke Math. J. 44 (1977) 329-355.

3. B. Blackadar, K-theory for operator algebras, Mathematical Sciences Research Institute Publications 5 (Springer, New York, 1986).

4. O. Bratteli, 'Inductive limits of finite dimensional $\mathrm{C}^{*}$-algebras', Trans. Amer. Math. Soc. 171 (1972) 195-234. 
5. L. G. Brown, 'Stable isomorphism of hereditary subalgebras of $\mathrm{C}^{*}$-algebras', Pacific J. Math. 71 (1977) 335-348.

6. L. G. Brown, R. G. Douglas and P. A. Fillmore, 'Extensions of $\mathrm{C}^{*}$-algebras and K-homology', Ann. of Math. 105 (1977) 265-324.

7. L. G. Brown and G. K. Pedersen, 'C ${ }^{*}$-algebras of real rank zero', J. Funct. Anal. 99 (1991) $131-149$.

8. A. Connes and N. Higson, 'Déformations, morphismes asymptotiques et $K$-théorie bivariante', C. R. Acad. Sci. Paris Sér. I Math. 311 (1990) 101-106.

9. J. CunTz, 'The structure of multiplication and addition in simple $\mathrm{C}^{*}$-algebras', Math. Scand. 40 (1977) 215-233.

10. J. CunTZ, ' $K$-theory for certain $C^{*}$-algebras', Ann. of Math. 113 (1981) 181-197.

11. J. CunTz, 'Generalized homomorphisms between $\mathrm{C}^{*}$-algebras and $K K$-theory', Dynamics and processes (ed. P. Blanchard and L. Streit), Lecture Notes in Mathematics 1031 (Springer, Berlin, 1983) 31-45.

12. M. Dadarlat, 'Quasidiagonal morphisms and homotopy', J. Funct. Anal. 151 (1997) 213-233.

13. M. Dadarlat, 'Nonnuclear subalgebras of AF algebras', Amer. J. Math. 122 (2000) 581-597.

14. M. Dadarlat and S. Eilers, 'Asymptotic unitary equivalence in $K K$-theory', $K$-theory 23 (2001) 305-322.

15. M. Dadarlat and G. Gong, 'A classification result for approximately homogeneous $\mathrm{C}^{*}$-algebras of real rank zero', Geom. Funct. Anal. 7 (1997) 646-711.

16. M. Dadarlat and T. A. Loring, 'The $K$-theory of Abelian subalgebras of AF algebras', $J$. reine angew. Math. 432 (1992) 39-55.

17. M. Dadarlat and T. A. Loring, 'Extensions of certain real rank zero $\mathrm{C}^{*}$-algebras', Ann. Inst. Fourier 44 (1994) 907-925.

18. M. Dadarlat and T. A. Loring, 'Classifying $\mathrm{C}^{*}$-algebras via ordered, mod- $p$-theory', Math. Ann. 305 (1996) 601-616.

19. M. Dadarlat and T. A. Loring, 'A universal multicoefficient theorem for the Kasparov groups', Duke Math. J. 84 (1996) 355-377.

20. S. EILERS and T. A. Loring, 'Computing contingencies for stable relations', Internat. J. Math. 10 (1999) 301-326.

21. G. A. Elliott, 'On the classification of inductive limits of sequences of semisimple finitedimensional algebras', J. Algebra 38 (1976) 29-44.

22. G. A. Elliott, 'On the classification of $\mathrm{C}^{*}$-algebras of real rank zero', J. reine angew. Math. 443 (1993) 179-219.

23. G. A. Elliott and G. Gong, 'On the classification of $\mathrm{C}^{*}$-algebras of real rank zero, II', Ann. of Math. 144 (1996) 497-610.

24. L. Fuchs, Infinite abelian groups I (Academic Press, New York, 1970).

25. N. Higson, 'On a technical theorem of Kasparov', J. Funct. Anal. 73 (1987) 107-112.

26. A. Hulanicki, 'On algebraically compact groups', Bull. Acad. Polon. Sci. Sér. Sci. Math. Astronom. Phys. 10 (1962) 71-75.

27. A. HulanicKI, 'The structure of the factor group of the unrestricted sum by the restricted sum of Abelian groups', Bull. Acad. Polon. Sci. Sér. Sci. Math. Astronom. Phys. 10 (1962) 77-80.

28. K. K. Jensen and K. Thomsen, Elements of KK-theory (Birkhäuser, Boston, 1991).

29. G. G. Kasparov, 'Hilbert $C^{*}$-modules: theorems of Stinespring and Voiculescu', J. Operator Theory 4 (1980) 133-150.

30. E. KirchberG, 'The classification of purely infinite $\mathrm{C}^{*}$-algebras using Kasparov's theory', Preprint, 1994.

31. E. Kirchberg and N. C. Phillips, 'Embedding of exact $\mathrm{C}^{*}$-algebras in the Cuntz algebra $\mathcal{O}_{2}$ ', J. reine angew. Math. 525 (2000) 17-53.

32. H. Lin, 'Exponential rank of $\mathrm{C}^{*}$-algebras with real rank zero and the Brown-Pedersen conjectures', J. Funct. Anal. 114 (1993) 1-11.

33. H. LIN, 'Approximation by normal elements with finite spectra in $\mathrm{C}^{*}$-algebras of real rank zero', Pacific J. Math. 173 (1996) 443-489.

34. H. Lin, 'Stable approximately unitary equivalence of homomorphisms', J. Operator Theory to appear.

35. H. Lin, 'Tracially AF C*-algebras', Trans. Amer. Math. Soc. 353 (2001) 693-722.

36. H. LIN, 'Classification of simple tracially AF C*-algebras', Canad. J. Math. 53 (2001) 161-194.

37. H. X. Lin and N. C. Phillips, 'Almost multiplicative morphisms and the Cuntz algebra $\mathcal{O}_{2}$ ', Internat. J. Math. 6 (1995) 625-643.

38. T. A. LORING, Lifting solutions to perturbing problems in $C^{*}$-algebras, Fields Institute Monographs 8 (American Mathematical Society, Providence, RI, 1997).

39. N. C. Phillips, 'Exponential length and traces', Proc. Roy. Soc. Edinburgh Sect. A 125 (1995) $13-29$.

40. N. C. Phillips, 'Approximate unitary equivalence of homomorphisms from odd Cuntz algebras', 
Operator algebras and their applications, Waterloo, ON, 1994/1995 (ed. P. A. Fillmore and J. A. Mingo), Fields Institute Communications 13 (American Mathematical Society, Providence, RI, 1997) 243-255.

41. N. C. Phillips, 'A classification theorem for nuclear purely infinite simple $\mathrm{C}^{*}$-algebras', Doc. Math. 5 (2000) 49-114.

42. S. PopA, 'On local finite-dimensional approximation of $\mathrm{C}^{*}$-algebras', Pacific J. Math. 181 (1997) $141-158$

43. M. A. RIEFFEL, 'Dimension and stable rank in the $K$-theory of $\mathrm{C}^{*}$-algebras', Proc. London Math. Soc. (3) 46 (1983) 301-333.

44. M. A. RIEFFEL, 'The homotopy groups of the unitary groups of noncommutative tori', J. Operator Theory 17 (1987) 237-254.

45. M. RøRDAM, 'Classification of inductive limits of Cuntz algebras', J. reine angew. Math. 440 (1993) 175-200.

46. M. RøRDAM, 'Classification of certain infinite simple $C^{*}$-algebras', J. Funct. Anal. 131 (1995) $415-458$.

47. J. Rosenberg and C. Schochet, 'The Künneth theorem and the universal coefficient theorem for Kasparov's generalized $K$-functor', Duke Math. J. 55 (1987) 431-474.

48. N. Salinas, 'Relative quasidiagonality and $K K$-theory', Houston J. Math. 18 (1992) 97-116.

49. C. Schоснет, 'Topological methods for $C^{*}$-algebras IV: Mod $p$ homology', Pacific J. Math. 114 (1984) 447-468.

50. C. L. Schoснет, 'On the fine structure of the Kasparov groups I: continuity of the $K K$-pairing', J. Funct. Anal. 186 (2001) 25-61.

51. G. Skandalis, 'Une notion de nucléarité en $K$-théorie (d'après J. Cuntz)', K-Theory 1 (1988) 549-573.

52. S. Zhang, 'A property of purely infinite simple C*-algebras', Proc. Amer. Math. Soc. 109 (1990) 717-720.

53. S. Zhang, 'Matricial structure and homotopy type of simple $\mathrm{C}^{*}$-algebras with real rank zero', J. Operator Theory 126 (1991) 283-312.

\author{
Marius Dadarlat \\ Department of Mathematics \\ Purdue University \\ West Lafayette \\ IN 47907 \\ USA
}

mdd@math.purdue.edu

\author{
Søren Eilers \\ Matematisk Afdeling \\ Kobenhavns Universitet \\ Universitetsparken 5 \\ DK-2100 Copenhagen $\varnothing$ \\ Denmark
}

eilers@math.ku.dk 